\title{
A universal Lipschitz extension property of Gromov hyperbolic spaces
}

\section{Alexander Brudnyi and Yuri Brudnyi}

\begin{abstract}
A metric space $U$ has the universal Lipschitz extension property if for an arbitrary metric space $M$ and every subspace $S$ of $M$ isometric to a subspace of $U$ there exists a continuous linear extension of Banach-valued Lipschitz functions on $S$ to those on all of $M$. We show that the finite direct sum of Gromov hyperbolic spaces of bounded geometry is universal in the sense of this definition.
\end{abstract}

\section{Formulation of Main Results}

In order to present a precise formulation of the main results we need several definitions.

Let $(M, d)$ be a metric space with underlying set $M$ and metric $d$ (we write simply $M$ if $d$ can be restored from the context). The space of Banach-valued Lipschitz functions on $M$ with target space $X$ is denoted by $\operatorname{Lip}(M, X)$; this space is endowed with the standard seminorm

$$
L(f):=\sup _{m \neq m^{\prime}}\left\{\frac{\left\|f(m)-f\left(m^{\prime}\right)\right\|}{d\left(m, m^{\prime}\right)}\right\} .
$$

(In case $X=\mathbb{R}$ we write $\operatorname{Lip}(M)$ instead of $\operatorname{Lip}(M, \mathbb{R})$.)

A subset $S \subset M$ will be regarded as a metric (sub-) space equipped with the induced metric $\left.d\right|_{S \times S}$. Hence, the notations $\operatorname{Lip}(S, X)$, and $L(f)$ for $f \in \operatorname{Lip}(S, X)$ are clear.

A simultaneous Lipschitz extension from $S$ to $M$ is a continuous linear operator $T: \operatorname{Lip}(S, X) \rightarrow \operatorname{Lip}(M, X)$ such that

$$
\left.T f\right|_{S}=f .
$$

2000 Mathematics Subject Classification: Primary 26B35, Secondary 54E35, 46B15. Keywords: Metric space, Lipschitz function, linear extension. 
The set of all such $T$ is denoted by $\operatorname{Ext}(S, M ; X)$ and an (optimal) extension constant is given by

$$
\lambda(S, M ; X):=\inf \{\|T\|: T \in \operatorname{Ext}(S, M ; X)\} .
$$

(This becomes $\infty$, if $\operatorname{Ext}(S, M ; X)=\emptyset$.)

It is shown in $[\mathrm{BB}]$ that there are rather simple metric spaces (e.g., metric graphs with the vertex degrees bounded by 3 ) and subspaces of these spaces for which $\operatorname{Ext}(S, M ; \mathbb{R})=\emptyset$. The results presented below show that nevertheless there are many subspaces in a metric space for which the extension constants (1.2) are finite.

In what follows we will use the following definitions.

A map $\phi:(M, d) \rightarrow\left(M_{1}, d_{1}\right)$ is said to be C-Lipschitz, if its Lipschitz constant is bounded by a constant $C$ (and simply Lipschitz, if $L(\phi)$ is bounded).

If, in addition, $\phi$ is an injection and for all $m, m^{\prime} \in M$ and given $C \geq 1$

$$
C^{-1} d\left(m, m^{\prime}\right) \leq d_{1}\left(\phi(m), \phi\left(m^{\prime}\right)\right) \leq C d\left(m, m^{\prime}\right)
$$

then $\phi$ is a $C$-isometric embedding (simply quasi-isometric embedding, if (1.3) holds for some $C$ ).

Note that the distortion of $\phi($ written $\operatorname{dst}(\phi))$ satisfies

$$
\operatorname{dst}(\phi):=L(\phi) L\left(\phi^{-1}\right) \leq C^{2} .
$$

Finally, $\phi: M \rightarrow M_{1}$ is a $C$-isometry, if $\phi$ is a bijection satisfying (1.3). (A 1-isometry is called isometry.)

Now we present the basic definitions of the paper.

Definition 1.1 A metric space $U$ is said to be universal with respect to simultaneous Lipschitz extensions if for an arbitrary metric space $M$ and every subspace $S$ of $M$ isometric to a subspace of $U$

$$
\lambda(S, M ; X) \leq c(U)
$$

where $c(U)$ depends only on $U$.

Remark 1.2 In fact, in all our results related to universality we will establish a stronger property: if, using the notation of Definition 1.1, $S$ is $C$-isometric $(C \geq 1)$ to a subspace of $U$, then

$$
\lambda(S, M ; X) \leq C^{2} c(U)
$$

with $c(U)$ depending only on $U$. This clearly implies the universality of $U$. 
A deep result by Lee and Naor, see [LN, Theorem 1.6], implies universality in this sense of every doubling metric space. Let us recall that $M$ is doubling if there is a constant $D$ such that for each $R>0$ every ball of radius $2 R$ can be covered by at most $D$ balls of radius $R$. The minimal $D$ is the doubling constant of $M$ (denoted by $D(M)$ ).

The aforementioned theorem states that if $M_{0}$ is a doubling subspace of a metric space $M$, then for some numerical constant $C \geq 1$

$$
\sup _{X} \lambda\left(M_{0}, M ; X\right) \leq C \log _{2} D\left(M_{0}\right) \text {. }
$$

Since every subspace $S$ of $M_{0}$ inherits the doubling property with $D(S) \leq$ $D\left(M_{0}\right)$, inequality (1.5) implies the universality of $M_{0}$.

The main result of this paper presents a wide class of universal metric spaces which have no such hereditary property. For its formulation we require

Definition 1.3 A metric space is of bounded geometry with parameters $n$, $R, C$ if every open ball of this space of radius $R$ admits a $C$-isometric embedding into $\mathbb{R}^{n}$.

Remark 1.4 Hereafter $\mathbb{R}^{n}$ is regarded as the Euclidean space endowed with the standard norm $\|x\|_{2}:=\left\{\sum_{i=1}^{n} x_{i}^{2}\right\}^{1 / 2}, x=\left(x_{1}, \ldots, x_{n}\right)$.

Notation. The class of metric spaces, satisfying Definition 1.3 is denoted by $\mathcal{G}_{n}(R, C)$.

Our main result is

Theorem 1.5 Let $M:=\bigoplus_{i=1}^{N} M_{i}$ where every metric space $\left(M_{i}, d_{i}\right)$ is a (Gromov) hyperbolic space of bounded geometry. Then $M$ is universal.

Here $\bigoplus_{i=1}^{N} M_{i}$ is a metric space with underlying set $\prod_{i=1}^{N} M_{i}$ and metric $d:=\max _{1 \leq i \leq N} d_{i}$. In the sequel we also use direct p-products of these spaces $(1 \leq p \leq \infty)$ with the same underlying set and the metric $d_{p}$ given by

$$
d_{p}\left(m, m^{\prime}\right):=\left(\sum_{i=1}^{N} d_{i}^{p}\left(m_{i}, m_{i}^{\prime}\right)\right)^{1 / p}
$$

For the convenience of the reader we recall the Rips definition of Gromov hyperbolicity (the background material, basic properties and examples can be found in the monographs $[\mathrm{BH}]$ and $[\mathrm{Gr}])$. 
Definition 1.6 A geodesic metric space is $\delta$-hyperbolic $(\delta \geq 0)$ if every geodesic triangle is $\delta$-slim, that is, each side of the triangle lies in the $\delta$ neighbourhood of the union of the remaining sides.

We will say that $M$ is (Gromov) hyperbolic if it is $\delta$-hyperbolic for some $\delta \geq 0$.

Let us also recall that a metric space $(M, d)$ is said to be geodesic, if every pair of points can be joined by a geodesic segment. In turn, a geodesic segment joining $m$ and $m^{\prime}$ is the image of a geodesic, a curve $\gamma:[0, a] \rightarrow M$ such that $\gamma(0)=m, \gamma(a)=m^{\prime}$ and $d(\gamma(t), \gamma(s))=|t-s|$ for all $t, s \in[0, a]$ (in particular, $a=d\left(m, m^{\prime}\right)$ and also is equal to the length of $\gamma$ ).

Finally, a geodesic triangle with vertices $m_{1} \neq m_{2} \neq m_{3}$ is the union of geodesic segments with endpoints at these points.

Example 1.7 (a) The Lobachevski space $\mathbb{H}^{n}$ is $\delta$-hyperbolic with $\delta=\ln 3$, see, e.g., $[\mathrm{CDP}]$. A straightforward computation also shows that $\mathbb{H}^{n}$ is of bounded geometry and belongs to $\mathcal{G}_{n}(R, C)$ for every $R>0$ and $C=C(n, R){ }^{1}$

(b) A simply connected $n$-dimensional Riemannian manifold with sectional curvature $\kappa$ satisfying $-b^{2} \leq \kappa \leq-a^{2}<0$ for some $a, b>0$, is hyperbolic (a consequence of (a) and Toponogov's comparison theorem) and belongs to $\mathcal{G}_{n}(R, C)$ with $C=C(n, R)$ for every $R>0$ (a consequence of Rauch's comparison theorem).

(c) A metric tree is 0-hyperbolic, since there are no nondegenerate triangles (cycles) in this space. If the degrees of the vertices of the tree are uniformly bounded, it belongs to $\mathcal{G}_{2}(R, C), C=C(R)$, for any $R>0$.

(d) A bounded strongly pseudoconvex domain in $\mathbb{C}^{n}$ with the Kobayashi metric is Gromov hyperbolic and of bounded geometry. More generally this holds for uniform domains in $\mathbb{R}^{n}$ with the quasi-hyperbolic metric, see [BHK, Chapter 1].

Remark 1.8 Theorem 1.5 is of interest only in the case of unbounded geodesic spaces $M_{i}$. In fact, a bounded geodesic space is clearly Gromov hyperbolic. If, in addition, it is of bounded geometry, then by Proposition 2.2 below this space is doubling and its universality follows from the Lee-Naor theorem.

Combining Theorem 1.5 with the above mentioned result of Lee and Naor [LN, Theorem 1.6] one obtains the following

Corollary 1.9 Let $M:=\bigoplus_{i=1}^{N} M_{i}$ where every $\left(M_{i}, d_{i}\right)$ is either a doubling metric space or a Gromov hyperbolic space of bounded geometry. Then $M$ is universal.

\footnotetext{
${ }^{1}$ In the sequel $C, C_{1}$ etc denote constants; we write $C=C(\alpha, \beta, \ldots)$ if the $C$ depends only on the parameters $\alpha, \beta, \ldots$.
} 
The proof of Theorem 1.5 is based on several recent results on Lipschitz embeddings and extensions and a new theorem of our own that will be formulated now. For this goal we need several notions.

Definition 1.10 A nonnegative Borel measure on a metric space $(M, d)$ is said to be doubling at a point $m \in M$ if every open ball centered at $m$ is of finite strictly positive measure and the doubling constant

$$
D_{m}(\mu):=\sup _{R>0} \frac{\mu\left(B_{2 R}(m)\right)}{\mu\left(B_{R}(m)\right)}<\infty .
$$

If, in addition,

$$
D(\mu):=\sup _{m \in M} D_{m}(\mu)<\infty
$$

then $\mu$ is said to be a doubling measure.

Here and below $B_{R}(m)$ is the open ball $\left\{m^{\prime} \in M: d\left(m, m^{\prime}\right)<R\right\}$ and $\bar{B}_{R}(m)$ is the closed ball $\left\{m^{\prime} \in M: d\left(m, m^{\prime}\right) \leq R\right\}$.

A metric space endowed with a fixed doubling measure is said to be of homogeneous type; that is to say, this is a triple $(M, d, \mu)$ where $\mu$ is a doubling measure. It is known, see $[\mathrm{CW}]$, that

$$
\log _{2} D(M) \leq c \log _{2} D(\mu)
$$

where $c>1$ is a numerical constant.

The following definition gives a useful generalization of spaces of homogeneous type.

Definition 1.11 A metric space $(M, d)$ with a fixed family of nonnegative Borel measures $\left\{\mu_{m}\right\}_{m \in M}$ on $M$ is said to be of pointwise homogeneous type if the following holds.

(i) Uniform doubling condition:

For every $m \in M, \mu_{m}$ is doubling at $m$ and

$$
D:=\sup _{m \in M} D_{m}\left(\mu_{m}\right)<\infty .
$$

(ii) Consistency with the metric:

For some constant $C>0$ and all $m_{1}, m_{2} \in M$ and $R>0$ such that $d\left(m_{1}, m_{2}\right) \leq R$

$$
\left|\mu_{m_{1}}-\mu_{m_{2}}\right|\left(B_{R}(m)\right) \leq C \frac{\mu_{m}\left(B_{R}(m)\right)}{R} d\left(m_{1}, m_{2}\right)
$$

where $m=m_{1}$ or $m_{2}$.

By $C(t) \leq C, 0 \leq t \leq 1$, we denote the optimal constant in the above inequality with $d\left(m_{1}, m_{2}\right) \leq t R, m_{1}, m_{2} \in M$. 
The next examples clarify this definition.

Example 1.12 (a) A metric space $(M, d)$ of homogeneous type endowed with a doubling measure $\mu$ clearly satisfies Definition 1.11 with $C=0$ and $D=D(\mu)$.

(b) Let $(M, d)$ be a doubling metric space (with doubling constant $D(M)$ ). By the Koniagin-Vol'berg theorem [KV] (see also [LS]) $M$ carries a doubling measure $\mu$ such that

$$
\log _{2} D(\mu) \leq c \log _{2} D(M)
$$

with some numerical constant $c \geq 1$. Hence $(M, d)$ is of homogeneous type.

(c) $\mathbb{H}^{n}$ can be equipped with a family of Borel measures satisfying the conditions of Definition 1.11, see [BSh, pp. 537-540].

Finally, we need

Definition 1.13 A family of nonnegative Borel measures $\left\{\mu_{m}\right\}_{m \in M}$ on a metric space $M$ is said to be $K$-uniform $(K \geq 1)$, if for all $m_{1}, m_{2} \in M$ and $R>0$

$$
\mu_{m_{1}}\left(B_{R}\left(m_{1}\right)\right) \leq K \mu_{m_{2}}\left(B_{R}\left(m_{2}\right)\right)
$$

Now, all is ready to formulate our second main result. In its formulation $\left(M, d_{p}\right)$ is the direct $p$-sum $\bigoplus_{i=1}^{N}\left(M_{i}, d_{i}\right)$, see $(1.6)$, and $\left(M_{i}, d_{i}\right)$ is of pointwise homogeneous type with respect to a family of Borel measures $\left\{\mu_{m}^{i}\right\}_{m \in M_{i}}$ with optimal constants $D_{i}$ and $C_{i}:=C_{i}(1), 1 \leq i \leq N$, see Definition 1.11.

Theorem 1.14 Assume that $\left\{\mu_{m}^{i}\right\}_{m \in M_{i}}$ is $K_{i}$-uniform for all $1 \leq i \leq N$. Then for every subspace $S \subset\left(M, d_{p}\right)$ the extension constant, see (1.2), satisfies the following inequality:

$$
\lambda(S, M ; X) \leq c_{0}\left(\widetilde{C}_{p}+1\right)\left(\log _{2} \widetilde{D}+1\right)
$$

where

$$
\widetilde{D}:=\prod_{i=1}^{N} D_{i}, \quad \widetilde{C}_{p}:=\left(\sum_{i=1}^{N} C_{i}^{q}\right)^{1 / q} \prod_{i=1}^{N} K_{i}
$$

$c_{0}$ is a numerical constant and $q$ relates to $p$ by $\frac{1}{p}+\frac{1}{q}=1$.

For $X=\mathbb{R}$ a similar result is proved in the authors paper [BB, Theorem 2.25]. As an easy consequence one derives from there a special case of Theorem 1.14 when the target space $X$ is complemented in its second dual $X^{* *}$. In particular, the result holds for dual Banach spaces $X$ (i.e., $X=Y^{*}$ for a Banach space $Y$ ). But for general $X$ the proof of Theorem 2.25 presented in $[\mathrm{BB}]$ needs to be modified. This matter will be discussed in section 3 . 
Remark 1.15 (a) It is shown in the proof that for $N=1$ a sharper inequality holds:

$$
\lambda(S, M ; X) \leq c_{0}\left(C_{1}+1\right)\left(\log _{2} D_{1}+1\right)
$$

where $c_{0}$ is a numerical constant. In this case, the assumption of $K$-uniformity for the families $\left\{\mu_{m}^{i}\right\}$ is excluded from the theorem. This assumption can be eliminated also in the case $N>1$. Since this requires some additional technical consideration and enlarges substantially the right-hand side in (1.7), we will not state this generalization of Theorem 1.14.

(b) It is important for some applications that the extension operator of Theorem 1.14 maps a Lipschitz function $f: S \rightarrow X$ into a function whose range is contained in the closure of conv $f(S)$, the convex hull of $f(S)$. In particular, all the above formulated results are also true when the target space is a closed convex subset of a Banach space $X$.

(c) It can be seen from the proof that Theorem 1.14 remains true for $M_{1}$ a space of homogeneous type (with a doubling measure $\mu$ ). In this case $D_{1}=D(\mu), C_{1}=0$ and we may replace $K_{1}$ by 1 in (1.7), see Remarks 3.11 and 3.14 below.

\section{Proofs of Theorem 1.5 and Corollary 1.9}

Proof of Theorem 1.5. We need several auxiliary results. In the forthcoming formulations, a subset of $M$ is said to be $\epsilon-$ dense if its distance ${ }^{2}$ from each point of $M$ is less than $\epsilon$, and $\epsilon$-separated if the distance between every two distinct points of the set is more than or equal to $\epsilon$.

Proposition 2.1 (see [NPSS, Corollary 6.2]) Let $F: M \longrightarrow M_{1}$ be a $C$ Lipschitz map, and $A$ be an $\epsilon$-dense subset of $M$. Assume that there exists $\mu \in(0,1]$ such that for all $a, a^{\prime} \in A$

$$
d_{1}\left(F(a), F\left(a^{\prime}\right)\right) \geq \mu C d\left(a, a^{\prime}\right)
$$

Assume also that $M \in \mathcal{G}_{n_{0}}\left(R_{0}, C_{0}\right)$ and that

$$
\mu R_{0}=64 \epsilon
$$

Then there exist a constant $K=K\left(n_{0}, C_{0}, R_{0}, \mu, C\right)$ and an integer $N=N\left(n_{0}, C_{0}\right)$ such that $M$ admits a $K$-isometric embedding into the direct sum $M_{1} \oplus \mathbb{R}^{N}$.

\footnotetext{
${ }^{2}$ The distance from $S \subset M$ to $m$ is defined by $d(m, S):=\inf \left\{d\left(m, m^{\prime}\right): m^{\prime} \in S\right\}$.
} 
Proposition 2.2 Let $(M, d)$ be a geodesic metric space belonging to $\mathcal{G}_{n_{0}}\left(R_{0}, C_{0}\right)$. Then for every $R>0$ there exist an integer $n$ and a constant $C$ such that $M \in \mathcal{G}_{n}(R, C)$.

Proof. We must prove that every ball $B_{R}(m)$ admits a $C$-isometric embedding into some $\mathbb{R}^{n}$ where $C$ and $n$ are independent of the center $m$. To find the required embedding we choose a maximal $\epsilon$-separated set $A_{\epsilon}$ in $M$. Due to maximality, the family of balls $B_{a}:=B_{\epsilon}(a), a \in A_{\epsilon}$, covers $M$. On the other hand, the family $\widetilde{B}_{a}:=B_{\epsilon / 2}(a), a \in A_{\epsilon}$, consists of pairwise disjoint balls.

Lemma 2.3 (a) If $\epsilon \leq R_{0} / 2$, then the order of the open cover $\left\{B_{a}\right\}_{a \in A_{\epsilon}}$ is at most $\left(4 C_{0}^{2}\right)^{n_{0}}$.

(b) For the same $\epsilon$ and every $a \in A_{\epsilon}$ there is a bounded linear extension operator $E_{a}: \operatorname{Lip}\left(B_{a} \cap A_{\epsilon}\right) \rightarrow \operatorname{Lip}\left(B_{a}\right)$ whose norm is bounded by $24 n_{0} C_{0}^{2}$.

Proof. (a) By definition the order of $\mathcal{B}:=\left\{B_{a}\right\}_{a \in A_{\epsilon}}$ is given by

$$
\operatorname{ord}(\mathcal{B}):=\sup _{m \in M} \operatorname{card}\left\{a \in A_{\epsilon}: m \in B_{a}\right\} .
$$

The union $\cup\left\{B_{a}: B_{a} \ni m\right\}$ is contained in the ball $B_{2 \epsilon}(m)$. As $2 \epsilon \leq R_{0}$, there is a $C_{0}$-isometric embedding $\phi$ of $B_{2 \epsilon}(m)$ into the Euclidean ball $B_{\rho}(\phi(m)) \subset \mathbb{R}^{n_{0}}$ of radius $\rho:=2 C_{0} \epsilon$. On the other hand, the family $\left\{\phi\left(\widetilde{B}_{a}\right): a \in A_{\epsilon}\right\}$ consists of pairwise disjoint sets. This implies that that the family of Euclidean balls $\left\{B_{\rho^{\prime}}(\phi(a))\right\}_{B_{a} \ni m}, \rho^{\prime}:=\frac{\epsilon}{2 C_{0}}$, consists of pairwise disjoint sets containing in $B_{\rho}(\phi(m))$. Comparing the $n_{0}$-measures of the sets $\cup\left\{B_{\rho^{\prime}}(\phi(a)): B_{a} \ni m\right\}$ and $B_{\rho}(\phi(m))$ we then get

$$
\left(\frac{\epsilon}{2 C_{0}}\right)^{n_{0}} \operatorname{card}\left\{a \in A_{\epsilon}: B_{a} \ni m\right\} \leq\left(2 C_{0} \epsilon\right)^{n_{0}} .
$$

This implies the required estimate of $\operatorname{ord}(\mathcal{B})$.

(b) Since $B_{a} \subset B_{R_{0}}(a)$, there is a $C_{0}$-isometric embedding $\phi_{a}: B_{a} \rightarrow \mathbb{R}^{n_{0}}$. By the Whitney extension theorem there is a bounded linear extension operator acting from $\operatorname{Lip}\left(\phi_{a}\left(A_{\epsilon} \cap B_{a}\right)\right)$ into $\operatorname{Lip}\left(\mathbb{R}^{n_{0}}\right)$ whose norm is bounded by a constant $K=K\left(n_{0}\right)$; in [BB, Corollary 2.24] this constant is estimated by $24 n_{0}$. Then compositions with $\phi_{a}^{-1}$ and $\phi_{a}$ give the required operator $E_{a}: \operatorname{Lip}\left(A_{\epsilon} \cap B_{a}\right) \rightarrow \operatorname{Lip}\left(B_{a}\right)$.

Using an appropriate Lipschitz partition of unity subordinate to the cover $\left\{B_{a}: a \in A_{\epsilon} \cap B_{R}(m)\right\}$ of the ball $B_{R}(m)$ we paste together the operators $E_{a}$ to get a linear extension operator from $\operatorname{Lip}\left(A_{\epsilon} \cap B_{R}(m)\right)$ into $\operatorname{Lip}\left(B_{R}(m)\right)$ whose norm is bounded by a constant $k$ depending only on $\operatorname{ord}(\mathcal{B})$ and $\sup _{a}\left\|E_{a}\right\|$, see [BB, Lemma 11.3] for details. 
Then for the subspaces $\operatorname{Lip}_{0}\left(A_{\epsilon} \cap B_{R}(m)\right)$ and $\operatorname{Lip}_{0}\left(B_{R}(m)\right)$ of $\operatorname{Lip}\left(A_{\epsilon} \cap\right.$ $\left.B_{R}(m)\right)$ and $\operatorname{Lip}\left(B_{R}(m)\right)$ determined by the condition

$$
f\left(a^{*}\right)=0 \text { for a fixed } a^{*} \in A_{\epsilon} \cap B_{R}(m),
$$

we obtain the linear extension operator

$$
E: \operatorname{Lip}_{0}\left(A_{\epsilon} \cap B_{R}(m)\right) \rightarrow \operatorname{Lip}_{0}\left(B_{R}(m)\right) \text { with }\|E\| \leq k\left(n_{0}, C_{0}\right) .
$$

Now we use a duality argument which requires the Banach space $K(M)$ defined as the closed linear span in $\operatorname{Lip}_{0}(M)^{*}$ of the point evaluation functionals

$$
\delta_{M}(m)[f]:=f(m), \quad m \in M .
$$

By the Kantorovich-Rubinshtein duality theorem (see, e.g., [W] and references therein or the Appendix in $[\mathrm{BB}]$ )

$$
K(M)^{*}=\operatorname{Lip}_{0}(M) .
$$

Also, if $S \subset M$ is a subspace containing $a^{*}$, then by the McShane extension theorem (on extension of Lipschitz functions) $K(S)$ is naturally identified with a closed subspace of $K(M)$ and $\left.\delta_{M}\right|_{S}=\delta_{S}$.

We apply this construction to the spaces in (2.2). Since the domain of $E$ is finite-dimensional, there exists an operator

$$
P: K\left(B_{R}(m)\right) \rightarrow K\left(A_{\epsilon} \cap B_{R}(m)\right)
$$

such that

$$
P^{*}=E \quad \text { and } \quad\|P\|=\|E\| \leq k\left(n_{0}, C_{0}\right) .
$$

(By definition, $P$ is the restriction of the operator $E^{*}$ to $K\left(B_{R}(m)\right)$.) Moreover, $E$ is an extension operator and therefore $P$ is a linear projection onto $K\left(A_{\epsilon} \cap B_{R}(m)\right)$.

Next, by the McShane extension theorem

$$
\left\|\delta_{M}\left(m^{\prime}\right)-\delta_{M}\left(m^{\prime \prime}\right)\right\|_{K(M)}=d\left(m^{\prime}, m^{\prime \prime}\right), \quad m^{\prime}, m^{\prime \prime} \in M .
$$

In particular, $\delta_{B_{R}(m)}$ is an isometric embedding of $B_{R}(m)$ into $K\left(B_{R}(m)\right)$ and the analogous statement holds for $\delta_{B_{R}(m) \cap A_{\epsilon}}$.

Setting now $T:=P \circ \delta_{B_{R}(m)}$ we so define a $k\left(n_{0}, C_{0}\right)$-Lipschitz map of $B_{R}(m)$ into $K\left(A_{\epsilon} \cap B_{R}(m)\right)$ such that for $a^{\prime}, a^{\prime \prime} \in A_{\epsilon} \cap B_{R}(m)$ we have $\left\|T\left(a^{\prime}\right)-T\left(a^{\prime \prime}\right)\right\|_{K\left(A_{\epsilon} \cap B_{R}(m)\right)}=\left\|\delta_{B_{R}(m)}\left(a^{\prime}\right)-\delta_{B_{R}(m)}\left(a^{\prime \prime}\right)\right\|_{K\left(B_{R}(m)\right)}=d\left(a^{\prime}, a^{\prime \prime}\right)$.

Now we are under the conditions of Proposition 2.1 with $M:=B_{R}(m)$, $M_{1}:=K\left(A_{\epsilon} \cap B_{R}(m)\right), C:=k\left(n_{0}, C_{0}\right)$ and $\mu:=\frac{1}{k\left(n_{0}, C_{0}\right)}$ : choosing here $\epsilon$ equal to $\epsilon_{0}:=\frac{R_{0}}{64 k\left(n_{0}, C_{0}\right)}$ we derive from this proposition the following. 
There exist an integer $N=N\left(n_{0}, C_{0}\right)$ and a constant $k_{1}=k_{1}\left(n_{0}, C_{0}, R_{0}\right)$ such that the metric space $B_{R}(m)$ admits a $k_{1}$-isometric embedding into $K\left(A_{\epsilon_{0}} \cap B_{R}(m)\right) \bigoplus \mathbb{R}^{N}$.

Further, note that

$$
\operatorname{dim} K\left(A_{\epsilon_{0}} \cap B_{R}(m)\right)=\operatorname{card}\left(A_{\epsilon_{0}} \cap B_{R}(m)\right)-1:=d \leq N_{1}
$$

where $N_{1}$ is independent of the choice of $m$, see, e.g., [NPSS, page 18] for details. Also, $K\left(A_{\epsilon_{0}} \cap B_{R}(m)\right)^{*}$ is $C_{1}$-isometric to $l_{\infty}^{d}$ (considered as the space of bounded functions on $A_{\epsilon_{0}} \cap B_{R}(m)$ equal to 0 at $\left.a^{*}\right)$ with $C_{1}=C_{1}\left(\epsilon_{0}, R\right)$. This follows from the inequalities

$$
\left|f\left(a^{\prime}\right)-f\left(a^{\prime \prime}\right)\right| \leq 2\|f\|_{l_{\infty}^{d}} \leq \frac{2}{\epsilon_{0}}\|f\|_{l_{\infty}^{d}} d\left(a^{\prime}, a^{\prime \prime}\right), \quad a^{\prime}, a^{\prime \prime} \in A_{\epsilon_{0}} \cap B_{R}(m),
$$

and

$$
\|f\|_{l_{\infty}^{d}}:=\max _{a \in A_{\epsilon_{0}} \cap B_{R}(m)}|f(a)| \leq 2 L(f) R .
$$

Passing to the dual spaces we get from here that $K\left(A_{\epsilon_{0}} \cap B_{R}(m)\right)$ is $C_{1^{-}}$isometric to $l_{1}^{d}$. To finish the proof of the proposition it remains to use the natural linear quasi-isometry between $l_{1}^{d}$ and $l_{2}^{d}$ and the fact that $d \leq N_{1}$. Together with the previous statement this implies existence of a $C$-isometric embedding of $B_{R}(m)$ into $\mathbb{R}^{N+N_{1}}$ with $C=C\left(n_{0}, C_{0}, R_{0}, R\right)$.

Lemma 2.4 Let $(M, d)$ be the direct sum $\bigoplus_{i=0}^{N} M_{i}$ where $M_{i}=\mathbb{H}^{n_{i}}$ for $1 \leq i \leq N$, and $M_{0}$ is an $n_{0}$-dimensional Banach space. Then for the extension constant of $M$ we have

$$
\lambda(S, M, X) \leq c(M) .
$$

Proof. We apply Theorem 1.14 to our setting. (The proof of this theorem is presented in section 3.) In this case the Banach space $M_{0}$ endowed with the Lebesgue measure $\lambda_{M_{0}}$ is clearly of homogeneous type with parameters $D_{0}=2^{n_{0}}$ and $C_{0}=0$. Moreover,

$$
\lambda_{M_{0}}\left(B_{R}(m)\right)=c\left(n_{0}\right) R^{n_{0}}
$$

and therefore $\lambda_{M_{0}}$ is 1-uniform in the sense of Definition 1.13. Next, it was proved in [BSh, pp. 537-540] that there exist a metric $\rho_{i}$ on $M_{i}$ equivalent to the hyperbolic metric of $M_{i}$ and a family of Borel measures $\left\{\mu_{m}^{i}\right\}_{m \in M}$ such that $\left(M_{i}, \rho_{i}\right)$ is of pointwise homogeneous type with respect to this family, and, moreover, $\left\{\mu_{m}^{i}\right\}_{m \in M}$ is 1-uniform on $\left(M_{i}, \rho_{i}\right)$. Then the required result follows from Theorem 1.14. 
Lemma 2.5 Let $(M, d)$ be a Gromov hyperbolic space of bounded geometry. Then there are an integer $n$, a constant $K \geq 1$ and a finite-dimensional Euclidean space $B$ such that $M$ admits a K-isometric embedding into $\mathbb{H}^{n} \bigoplus B$.

Proof. By the Bonk-Schramm theorem [BS] there exists a rough $(C, k)$ similarity $\phi$ of $M$ into some $\mathbb{H}^{n}$ with constants $C \geq 1$ and $k \geq 0$. In other words, $\phi: M \rightarrow \mathbb{H}^{n}$ satisfies for all $m, m^{\prime}$

$$
C d\left(m, m^{\prime}\right)-k \leq d_{h}\left(\phi(m), \phi\left(m^{\prime}\right)\right) \leq C d\left(m, m^{\prime}\right)+k ;
$$

here $d_{h}$ is the inner metric on $\mathbb{H}^{n}$.

For $k=0$ this implies that $\phi$ is even a $C$-isometric embedding into $\mathbb{H}^{n}$. So it remains to consider the case $k>0$.

Set $\epsilon:=\frac{2 k}{C}$ and define $A \subset M$ to be a maximal $\epsilon$-separated set. That is, for all $a, a^{\prime} \in A$ with $a \neq a^{\prime}$

$$
d\left(a, a^{\prime}\right) \geq \epsilon
$$

and because of maximality for every $m \in M$ there is $a \in A$ such that $d(m, a)<\epsilon$. From (2.4), (2.5) and the choice of $\epsilon$

$$
\frac{C}{2} d\left(a, a^{\prime}\right) \leq d_{h}\left(\phi(a), \phi\left(a^{\prime}\right)\right) \leq \frac{3 C}{2} d\left(a, a^{\prime}\right)
$$

for all $a, a^{\prime} \in A$. Hence, $\left.\phi\right|_{A}$ is a $\frac{3 C}{2}$-Lipschitz map from $A$ into $\mathbb{H}^{n}$. By the Lang-Pavlović-Schroeder extension theorem [LPS] $\left.\phi\right|_{A}$ admits a Lipschitz extension $\widehat{\phi}: M \rightarrow \mathbb{H}^{n}$ with Lipschitz constant

$$
L(\widehat{\phi}) \leq \frac{3}{2} c(n) C .
$$

Moreover, at points $a, a^{\prime}$ of the $\epsilon$-net $A$ this map satisfies

$$
d_{h}\left(\widehat{\phi}(a), \widehat{\phi}\left(a^{\prime}\right)\right)=d_{h}\left(\phi(a), \phi\left(a^{\prime}\right)\right) \geq \frac{C}{2} d\left(a, a^{\prime}\right) .
$$

Finally, being a geodesic space of bounded geometry, $(M, d)$ belongs to $\mathcal{G}_{N}(R, C)$ for every $R>0$ and some $N, C$ depending only on $R$ and the parameters in the definition of bounded geometry for $M$, see Proposition 2.2. Choose

$$
R_{0}=\frac{384 k c(n)}{C}
$$

Then, the space $(M, d)$, the $\epsilon$-separated set $A$ and the Lipschitz map $\widehat{\phi}$ satisfy the conditions of Proposition 2.1. By this proposition there are a constant $K \geq 1$ and a finite-dimensional Euclidean space $B$ such that $(M, d)$ admits a $K$-isometric embedding into $\mathbb{H}^{n} \oplus B$. 
We are now ready to finish the proof of Theorem 1.5. So, let $S$ be a subspace of a metric space $\widehat{M}$ and let $\phi: S \rightarrow \bigoplus_{i=1}^{N} M_{i}$ be a $C$-isometric embedding. Here $M_{i}$ is a Gromov hyperbolic space of bounded geometry, $1 \leq i \leq N$.

We must find a linear extension operator $E: \operatorname{Lip}(S, X) \rightarrow \operatorname{Lip}(\widehat{M}, X)$ whose norm is bounded by a constant depending only on the characteristics of the spaces $M_{i}$ and the embedding constant $C(\geq L(\phi))$.

For this goal we first use Lemma 2.5 to a find a $C_{1}$-isometric embedding $\psi$ of $\bigoplus_{i=1}^{N} M_{i}$ into the space $\bigoplus_{i=1}^{N} \mathbb{H}^{n_{i}} \bigoplus \mathbb{R}^{n_{0}}$. Note that $C_{1}$ depends only on the characteristics of the spaces $M_{i}$. Then the composition $\psi \circ \phi$ is a $C C_{1}$-isometric embedding of $S$ into $\bigoplus_{i=1}^{N} \mathbb{H}^{n_{i}} \bigoplus \mathbb{R}^{n_{0}}$. Set

$$
\widehat{S}:=\operatorname{Image}(\psi \circ \phi) \subset\left(\bigoplus_{i=1}^{N} \mathbb{H}^{n_{i}} \bigoplus \mathbb{R}^{n_{0}}\right)
$$

and define the linear operator $E_{1}$ on $\operatorname{Lip}(S, X)$ by the formula

$$
E_{1} f:=f \circ \phi^{-1} \circ \psi^{-1} \text {. }
$$

Then $E_{1}: \operatorname{Lip}(S, X) \rightarrow \operatorname{Lip}(\widehat{S}, X)$ and

$$
\left\|E_{1}\right\| \leq C C_{1} .
$$

We use now Lemma 2.4 to find a linear continuous operator

$$
E_{2}: \operatorname{Lip}(\widehat{S}, X) \rightarrow \operatorname{Lip}\left(\bigoplus_{i=1}^{N} \mathbb{H}^{n_{i}} \bigoplus \mathbb{R}^{n_{0}}, X\right)
$$

such that

$$
\left.E_{2} g\right|_{\widehat{S}}=g \quad \text { for } \quad g \in \operatorname{Lip}(\widehat{S}, X)
$$

and, in addition,

$$
\left\|E_{2}\right\| \leq c(\bar{n})
$$

where $\bar{n}:=\left(n_{0}, n_{1}, \ldots, n_{N}\right)$.

Finally, the coordinatewise application of the Lang-Pavlović-Schroeder theorem [LPS] allows us to extend the map $\psi \circ \phi: S \rightarrow \bigoplus_{i=1}^{N} \mathbb{H}^{n_{i}} \bigoplus \mathbb{R}^{n_{0}}$ to a Lipschitz map $\Phi: \widehat{M} \rightarrow \bigoplus_{i=1}^{N} \mathbb{H}^{n_{i}} \bigoplus \mathbb{R}^{n_{0}}$ such that

$$
\left.\Phi\right|_{S}=\psi \circ \phi \quad \text { and } \quad L(\Phi) \leq c(n) C C_{1}
$$

where $n:=\sum_{i=0}^{n} n_{i}$. 
Next, define the linear operator $E_{3}$ on $\operatorname{Lip}\left(\bigoplus_{i=1}^{N} \mathbb{H}^{n_{i}} \bigoplus \mathbb{R}^{n_{0}}, X\right)$ by

$$
E_{3} h:=h \circ \Phi \text {. }
$$

Then $\operatorname{Lip}(\widehat{M}, X)$ is the target space of $E_{3}$ and

$$
\left\|E_{3}\right\| \leq L(\Phi) \leq c(n) C C_{1} .
$$

Moreover, by (2.10)

$$
\left.\left(E_{3} h\right)\right|_{S}=h\left(\left.\Phi\right|_{S}\right)=h \circ \psi \circ \phi
$$

Finally, define the desired linear extension operator $E$ by

$$
E=E_{3} E_{2} E_{1}
$$

According to $(2.6),(2.8)$ and $(2.12) E$ acts from $\operatorname{Lip}(S, X)$ into $\operatorname{Lip}(\widehat{M}, X)$ and

$$
\left.E f\right|_{S}=f .
$$

In addition, (2.7), (2.9) and (2.11) imply that

$$
\|E\| \leq C^{2} C_{1}^{2} C_{2}(\bar{n}) .
$$

Hence, the extension constant $\lambda(S, \widehat{M} ; X)$ is bounded by the constant on the right-hand side which depends only on the characteristics of the spaces $M_{i}$ and the embedding constant $C$ of $\phi$.

Proof of Corollary 1.9. Let $M:=\bigoplus_{i=1}^{N} M_{i}$. Without loss of generality we assume that $\left(M_{i}, d_{i}\right)$ is doubling for $i=1$ and Gromov hyperbolic of bounded geometry for $i \geq 2$.

Let $S$ be a subspace of an arbitrary metric space $\widetilde{M}$ and $\phi: S \rightarrow M$ be a $C$-isometric embedding. Set $M^{1}:=\bigoplus_{i=2}^{N} M_{i}$, so that $M=M_{1} \bigoplus M^{1}$.

By Lemma 2.5 we embed $M^{1}$ quasi-isometrically into

$$
H:=\bigoplus_{i=2}^{N} \mathbb{H}^{n_{i}} \bigoplus \mathbb{R}^{n_{0}} .
$$

Further, using the map $\delta_{M_{1}}$, see the proof of Proposition 2.2, we embed $M_{1}$ isometrically into the predual space $K\left(M_{1}\right)$ of $\operatorname{Lip}_{0}\left(M_{1}\right)$. The latter, in turn, we embed isometrically into the Banach space $l_{\infty}(B)$ where $B$ is the unit ball of $K\left(M_{1}\right)$. This allows us to identify the set $M$ with its image in $l_{\infty}(B) \bigoplus H$ and the map $\phi: S \rightarrow M$ with a quasi-isometric embedding into this image. Then $\phi=\left(\phi_{1}, \phi_{2}\right)$ where $\phi_{1}: S \rightarrow l_{\infty}(B)$ and $\phi_{2}: S \rightarrow H$. 
Next, by the McShane extension theorem, $\phi_{1}$ admits a Lipschitz extension to all of $\widetilde{M}$ preserving its Lipschitz constant while $\phi_{2}$ can be extended to all of $\widetilde{M}$ with Lipschitz constant bounded by $c\left(\sum_{i=2}^{N} n_{i}, n_{0}\right) L\left(\phi_{2}\right)$, by the Lang-Pavlović-Schroeder theorem [LPS]. Hence there is a Lipschitz map $\widetilde{\phi}: \widetilde{M} \rightarrow l_{\infty}(B) \bigoplus H$ such that $\left.\widetilde{\phi}\right|_{S}=\phi$ and $L(\widetilde{\phi})$ is bounded by a constant $c(M) C$.

Following the arguments of the proof of Theorem 1.5, we now determine certain linear extension operators $E_{1}: \operatorname{Lip}(\phi(S), X) \rightarrow \operatorname{Lip}\left(M_{1} \bigoplus H, X\right)$ and $E_{2}: \operatorname{Lip}\left(M_{1} \bigoplus H, X\right) \rightarrow \operatorname{Lip}\left(l_{\infty}(B) \bigoplus H, X\right)$ with bounds of their norms depending only on the basic parameters of $M$. Setting then

$$
E(f)[x]:=\left(E_{2} E_{1}\right)\left(f \circ \phi^{-1}\right)[\widetilde{\phi}(x)], \quad x \in \widetilde{M}, \quad f \in \operatorname{Lip}(S, X),
$$

we obtain a linear extension operator $\operatorname{Lip}(S, X) \rightarrow \operatorname{Lip}(\widetilde{M}, X)$ whose norm is bounded by the basic parameters of $M$ and $C$. This completes the proof of the corollary.

The operator $E_{1}$ is given by Theorem 1.14 with $M_{1}$ being a doubling metric space, see Remarks 3.11 and 3.14.

To define $E_{2}$ we first use the Lee-Naor bounded linear extension operator

$$
\widetilde{E}: \operatorname{Lip}\left(M_{1}, X\right) \rightarrow \operatorname{Lip}\left(l_{\infty}(B), X\right)
$$

whose norm is controlled by the doubling constant $D\left(M_{1}\right)$. Moreover, $\widetilde{E}$ is an averaging operator and therefore

$$
\left.\widetilde{E} f \subset \overline{\operatorname{conv} f\left(M_{1}\right)} \quad \text { (closure in } X\right) .
$$

Now for every $h \in H$ we define a linear operator

$$
\pi_{h}: \operatorname{Lip}\left(M_{1} \bigoplus H, X\right) \rightarrow \operatorname{Lip}\left(M_{1}, X\right)
$$

by $\pi_{h} f:=f(\cdot, h)$, and then set for $f \in \operatorname{Lip}\left(M_{1} \bigoplus H, X\right)$

$$
\left(E_{2} f\right)(m, h):=\left(\widetilde{E} \pi_{h} f\right)(m), \quad(m, h) \in l_{\infty}(B) \bigoplus H .
$$

By this definition

$$
\begin{aligned}
& \left\|\left(E_{2} f\right)\left(m_{1}, h_{1}\right)-\left(E_{2} f\right)\left(m_{2}, h_{2}\right)\right\|_{X} \\
& \leq\left\|\widetilde{E}\left(\pi_{h_{1}} f\right)\left(m_{1}\right)-\widetilde{E}\left(\pi_{h_{1}} f\right)\left(m_{2}\right)\right\|_{X}+\left\|\widetilde{E}\left[\left(\pi_{h_{1}}-\pi_{h_{2}}\right) f\right]\left(m_{2}\right)\right\|_{X} .
\end{aligned}
$$

The first term in the second line is bounded by $\|\widetilde{E}\| L(f) d_{M_{1}}\left(m_{1}, m_{2}\right)$ while the second one is bounded by

$$
\begin{aligned}
& \sup \left\{\|x\|_{X}: x \in \operatorname{conv}\left[f\left(\cdot, h_{1}\right)-f\left(\cdot, h_{2}\right)\right]\right\} \\
& =\sup \left\{\left\|\sum \alpha_{i}\left[f\left(m_{i}, h_{1}\right)-f\left(m_{i}, h_{2}\right)\right]\right\|_{X}: \alpha_{i} \geq 0, \sum \alpha_{i}=1 \text { and }\left\{m_{i}\right\} \subset M_{1}\right\} .
\end{aligned}
$$


This supremum is clearly bounded by $L(f) d_{H}\left(h_{1}, h_{2}\right)$. Together with the previous this gives the required estimate of the Lipschitz constant of $E_{2} f$ in $\operatorname{Lip}\left(l_{\infty}(B) \bigoplus H, X\right)$ by that of $f$.

\section{Proof of Theorem 1.14}

Let $S$ be a subset of a metric space $\left\{M, d_{p}\right\}$ where $M=\prod_{i=1}^{N} M_{i}$ and

$$
d_{p}:=\left\{\sum_{i=1}^{N} d_{i}^{p}\right\}^{1 / p} \quad(1 \leq p \leq \infty) .
$$

Let us recall that $\left(M_{i}, d_{i}\right)$ is of pointwise homogeneous type with respect to the family $\left\{\mu_{m}^{i}\right\}_{m \in M_{i}}$ of Borel measures on $M_{i}$, and $D_{i}, C_{i}$ are, respectively, the uniform doubling constant and the consistency constant for this family, see Definition 1.11. Moreover, the family $\left\{\mu_{m}^{i}\right\}_{m \in M_{i}}$ is $K_{i}$-uniform, see Definition 1.13.

Given these we must find a linear extension operator

$$
E: \operatorname{Lip}(S, X) \rightarrow \operatorname{Lip}(M, X)
$$

with the required estimate of its norm.

We divide the proof into three parts.

First, the required extension operator will be constructed for a single metric space of pointwise homogeneous type.

Then we will obtain the corresponding norm estimate for this operator.

Finally, the results obtained will be applied to prove the required result for the direct product of the spaces $M_{i}, 1 \leq i \leq N$.

\section{A. Extension operator}

Given a metric space $(M, d)$ of pointwise homogeneous type of Definition 1.11 and a subspace $S$ we now construct an extension operator $E$ acting from $\operatorname{Lip}(S, X)$ into $\operatorname{Lip}(M, X)$ and having the desired norm estimate. In the construction presented below $E$ acts between pointed Lipschitz spaces $\operatorname{Lip}_{0}(S, X)$ and $\operatorname{Lip}_{0}(M, X)$ determined by the condition

$$
f\left(m^{*}\right)=0 ;
$$

here $m^{*}$ is a fixed point in $S$. Since there exist linear projections on the pointed subspaces of norm one, the extension operator $E$ constructed for these subspaces gives rise to the required linear extension operator $\widehat{E}$ from $\operatorname{Lip}(S, X)$ into $\operatorname{Lip}(M, X)$ with $\|\widehat{E}\|=\|E\|$. 
We prefer to work with pointed Lipschitz spaces because of the following duality result which plays an essential role in our construction.

The space $K(M)=K(M, d)$ is defined to be the closed linear span of the point evaluation functionals

$$
\delta_{M}(m)(f):=f(m), \quad m \in M, \quad f \in \operatorname{Lip}_{0}(M),
$$

in $\operatorname{Lip}_{0}(M)^{*}$. Then the Kantorovich-Rubinshtein duality theorem states that

$$
K(M)^{*}=\operatorname{Lip}_{0}(M) .
$$

The map $\delta_{M}: M \rightarrow K(M)$ is readily seen to be an isometric embedding. Consider now the map

$$
\delta_{S}: S \rightarrow K(S)
$$

By the Dugundji extension theorem [D] there exists a continuous extension $\widehat{\delta}_{S}$ of $\delta_{S}$ to the whole of $M$ satisfying

$$
\widehat{\delta}_{S}(M) \subset \operatorname{span}\left(\delta_{S}(S)\right) .
$$

To apply this theorem we must assume that $S$ is closed. Clearly without loss of generality we can accept this restriction on $S$.

Let us recall Dugundji's extension construction.

Let $\left\{B_{m}\right\}_{m \in S^{c}}$ be an open cover of the open set $S^{c}:=M \backslash S$ by the open balls

$$
B_{m}:=B_{r_{m}}(m), \quad \text { where } \quad r_{m}:=\frac{1}{3} d(m, S) .
$$

Here the distance $d(m, S)$ from a point $m$ to $S$ is defined as $\inf _{m^{\prime} \in S} d\left(m, m^{\prime}\right)$.

Since any metric space is paracompact, there exists a continuous partition of unity $\left\{p_{\alpha}\right\}_{\alpha \in A}$ subordinate to the cover $\left\{B_{m}\right\}$ whose supports $U_{\alpha}:=\left\{m \in S^{c}: p_{\alpha}(m)>0\right\}$ form a locally finite cover of $S^{c}$.

For every $\alpha \in A$, we now pick points

$$
m_{1}(\alpha) \in S \quad \text { and } \quad m_{2}(\alpha) \in U_{\alpha}=\operatorname{supp} p_{\alpha}
$$

such that

$$
d\left(m_{1}(\alpha), m_{2}(\alpha)\right)<2 d\left(m_{2}(\alpha), S\right) .
$$

The aforementioned continuous extension $\widehat{\delta}_{S}$ is then given by

$$
\widehat{\delta}_{S}(m):=\left\{\begin{array}{clc}
\delta_{S}(m) & \text { if } & m \in S \\
\sum_{\alpha \in A} \delta_{S}\left(m_{1}(\alpha)\right) p_{\alpha}(m) & \text { if } & m \in S^{c} .
\end{array}\right.
$$


Lemma 3.1 Let $f \in \operatorname{Lip}_{0}(S, X)$. Then the function $\widehat{f}: M \rightarrow X$ given by

$$
\widehat{f}(m):=\left\{\begin{array}{ccc}
f(m) & \text { if } & m \in S \\
\sum_{\alpha \in A} f\left(m_{1}(\alpha)\right) p_{\alpha}(m) & \text { if } & m \in S^{c}
\end{array}\right.
$$

satisfies for all $m, m^{\prime} \in M$ the inequality

$$
\left\|\widehat{f}(m)-\widehat{f}\left(m^{\prime}\right)\right\|_{X} \leq 7 L(f)\left\{d\left(m, m^{\prime}\right)+d(m, S)+d\left(m^{\prime}, S\right)\right\} .
$$

Proof. In the case $m, m^{\prime} \in S$, inequality (3.8) (even with constant 1) is trivial, since $\widehat{f}=f$ on $S$ and $d(m, S)=d\left(m^{\prime}, S\right)=0$.

Let now $m \in S$ and $m^{\prime} \in S^{c}$. We denote by $V_{m}$ an open ball in the Banach space $X$ given by the inequality

$$
\|\widehat{f}(m)-x\|_{X}<\left(5 d\left(m, m^{\prime}\right)+2 d\left(m^{\prime}, S\right)\right) L(f), \quad x \in X .
$$

Inequality (3.8) in this case, clearly follows from the inclusion

$$
\widehat{f}\left(m^{\prime}\right) \in V_{m} .
$$

Since $\widehat{f}\left(m^{\prime}\right)$ is a convex combination of the points $f\left(m_{1}(\alpha)\right), \alpha \in A_{0}$, where the finite set $A_{0}$ is given by

$$
A_{0}:=\left\{\alpha \in A: m^{\prime} \in \operatorname{supp} p_{\alpha}\right\},
$$

see (3.7), inclusion (3.10) follows from the condition

$$
f\left(m_{1}(\alpha)\right) \in V_{m}, \quad \alpha \in A_{0} .
$$

This, in turn, is a consequence of the inequality

$$
d\left(m_{1}(\alpha), m\right)<5 d\left(m, m^{\prime}\right)+2 d\left(m^{\prime}, S\right)
$$

and the fact that $f \in \operatorname{Lip}_{0}(S, X)$.

To prove (3.11) we choose for $\alpha \in A_{0}$ a point $m(\alpha) \in S^{c}$ so that

$$
B_{m(\alpha)} \supset \operatorname{supp}_{\alpha} \quad\left(\ni m_{2}(\alpha)\right) .
$$

Then $m^{\prime} \in B_{m(\alpha)}, m \in S$, and this and (3.3) imply that $d(m(\alpha), S) \leq d(m(\alpha), m) \leq d\left(m(\alpha), m^{\prime}\right)+d\left(m^{\prime}, m\right) \leq \frac{1}{3} d(m(\alpha), S)+d\left(m, m^{\prime}\right)$.

Hence,

$$
d(m(\alpha), S) \leq d(m(\alpha), m) \leq \frac{3}{2} d\left(m, m^{\prime}\right)
$$

Further, $m_{2}(\alpha) \in B_{m(\alpha)}$ and therefore

$d\left(m_{2}(\alpha), m\right) \leq d\left(m_{2}(\alpha), m(\alpha)\right)+d(m(\alpha), m) \leq \frac{1}{3} d(m(\alpha), S)+d(m(\alpha), m)$. 
Combining this with the previous inequality we obtain

$$
d\left(m_{2}(\alpha), m\right) \leq 3 d\left(m, m^{\prime}\right) .
$$

Finally, this, (3.5) and (3.12) together with the inequality

$$
d\left(m_{1}(\alpha), m\right) \leq d\left(m_{1}(\alpha), m_{2}(\alpha)\right)+d\left(m_{2}(\alpha), m\right)
$$

give the required inequality (3.11).

It remains to consider the case of $m, m^{\prime} \in S^{c}$. Let, for the sake of definiteness,

$$
d\left(m^{\prime}, S\right) \leq d(m, S)
$$

Given $\epsilon>0$ we pick a point $m^{\prime \prime} \in S$ satisfying

$$
d\left(m^{\prime}, m^{\prime \prime}\right) \leq d\left(m^{\prime}, S\right)+\epsilon .
$$

We write now

$$
\left\|\widehat{f}(m)-\widehat{f}\left(m^{\prime}\right)\right\|_{X} \leq\left\|\widehat{f}(m)-\widehat{f}\left(m^{\prime \prime}\right)\right\|_{X}+\left\|\widehat{f}\left(m^{\prime \prime}\right)-\widehat{f}\left(m^{\prime}\right)\right\|_{X}
$$

Since $m^{\prime \prime} \in S$, we can apply the estimate obtained in the previous part of the proof to bound the right-hand side by

$$
L(f)\left\{2\left(d(m, S)+d\left(m^{\prime}, S\right)\right)+5\left(d\left(m, m^{\prime \prime}\right)+d\left(m^{\prime}, m^{\prime \prime}\right)\right)\right\} .
$$

Moreover, by the choice of $m^{\prime \prime}$

$$
d\left(m, m^{\prime \prime}\right) \leq d\left(m, m^{\prime}\right)+d\left(m^{\prime}, m^{\prime \prime}\right) \leq d\left(m, m^{\prime}\right)+d\left(m^{\prime}, S\right)+\epsilon .
$$

Therefore, the sum in the curly brackets is bounded by

$$
2\left(d(m, S)+d\left(m^{\prime}, S\right)\right)+5 d\left(m, m^{\prime}\right)+10 d\left(m^{\prime}, S\right)+10 \epsilon .
$$

This and (3.13), in turn, give the required inequality (3.8).

The lemma has been proved.

We are now ready to define the required extension operator $E$. It is given for $f \in \operatorname{Lip}_{0}(S, X)$ by

$$
(E f)(m):=\left\{\begin{array}{ccc}
f(m) & \text { if } & m \in S \\
I(\widehat{f} ; m, d(m)) & \text { if } & m \in S^{c} .
\end{array}\right.
$$

Here

$$
d(m):=d(m, S)
$$

$\widehat{f}$ is defined by (3.7), and for a locally continuous and locally bounded func$\operatorname{tion}^{3} g: M \rightarrow X$ we set

$$
I(g ; m, R):=\frac{1}{\mu_{m}\left(B_{R}(m)\right)} \int_{B_{R}(m)} g d \mu_{m} .
$$

\footnotetext{
${ }^{3}$ i.e., continuous and bounded on every bounded subset of $M$.
} 
Let us recall that $\left\{\mu_{m}\right\}_{m \in M}$ is the family of Borel measures on $M$, subject to Definition 1.11.

Let us show that $E$ is well-defined, that is, that the vector function $\widehat{f}$ is (strongly) continuous and bounded on every bounded subset of $M$.

Indeed, it is well-known (see, e.g., [GK]) that for any $f \in \operatorname{Lip}_{0}(S, X)$ there exists a uniquely defined linear continuous map $\tilde{f}: K(S) \rightarrow X$ such that

$$
f=\tilde{f} \circ \delta_{S}
$$

Then by the definitions of $\widehat{f}$, see (3.7), and $\widehat{\delta}_{S}$, see (3.6), we have

$$
\widehat{f}=\widetilde{f} \circ \widehat{\delta}_{S} .
$$

Since all the functions on the right-hand side are continuous and locally bounded, $\widehat{f}$ is continuous and locally bounded on $M$. Therefore the integral $I((\widehat{f} ; m, d(m))$ is finite.

Remark 3.2 Our construction of the operator $E$ would be much simpler if we could define a Borel measurable map $\phi: S^{c} \rightarrow S$ satisfying the condition

$$
d(m, \phi(m)) \leq C d(m, S), \quad m \in M
$$

with some constant $C \geq 1$ independent of $m$ and $S$. Then $\widehat{f}$ in (3.14) would be replaced by the composite $f \circ \phi$ for which an inequality similar to inequality (3.8) of Lemma 3.1 trivially holds (with 7 replaced by $C$ ).

Unfortunately, such $\phi$ does not exist in general even in the simplest case of $M=\mathbb{R}^{2}$, see the corresponding counter-example in the paper [N] by P. Novikov.

At the next stage we must estimate the norm of the constructed extension operator. The derivation presented below leads to an expression which contains $\max \left(\frac{l}{l-1}, D(l)\right)$ where the function $D:[1, \infty) \rightarrow \mathbb{R}_{+}$is given by

$$
D(l):=\sup _{m \in M} \sup _{R>0} \frac{\mu_{m}\left(B_{l R}(m)\right)}{\mu_{m}\left(B_{R}(m)\right)} .
$$

If $D(l)=l^{\lambda}$ for some constant $\lambda \geq 1$, then the term $\max \left(\frac{l}{l-1}, D(l)\right)$ can by minimized by choosing $l:=1+1 / \lambda$. This gives the bound $O(\lambda)=O\left(\log _{2} D\right)$ as required.

In general, we have for $l \leq 2$ only the trivial estimate $D(l) \leq D$ which does not allow to achieve the result declared in Theorem 1.14 (for $N=1$ ).

To overcome this obstacle we replace the original metric space $(M, d)$ by a new one for which the $D(l)$ is "almost" $l^{\lambda}$ for some $\lambda>1$. Moreover, this 
new space, say $(\widehat{M}, \widehat{d})$, contains an isometric copy of $(M, d)$. Therefore the extension constants of these spaces, see (1.2), satisfy

$$
\sup _{S \subset M} \lambda(S, M ; X) \leq \sup _{\widehat{S} \subset \widehat{M}} \lambda(\widehat{S}, \widehat{M} ; X) .
$$

As soon as an appropriate bound of the right-hand side of (3.18) via the doubling and the consistency constants for the $M$, has been obtained the desired inequality for $\lambda(S, M ; X)$ has been established. We will realize this program in two steps:

In subsection $\mathrm{B}$, we estimate the basic parameters of $\widehat{M}$ via those of $M$.

In the next subsection, we obtain the required estimate for the norm of extension operator (3.14). This gives the proof of Theorem 1.14 for a single space $(N=1)$.

\section{B. The basic properties of the extended metric space}

The desired metric space $(\widehat{M}, \widehat{d})$ has underlying set

$$
M_{n}:=M \times \mathbb{R}^{n}
$$

and metric given by

$$
d_{n}:=d \bigoplus \delta_{n}
$$

where $d$ is the metric of the original space $M$ and $\delta_{n}$ is the $l_{1}$-metric of $\mathbb{R}^{n}$. The integer $n \geq 2$ will be chosen later to minimize the corresponding estimates.

We then equip the space $\left(M_{n}, d_{n}\right)$ with the family of measures $\mathcal{F}_{n}:=$ $\left\{\mu_{\widetilde{m}}\right\}_{\widetilde{m} \in M_{n}}$ where

$$
\mu_{\tilde{m}}:=\mu_{m} \otimes \lambda_{n}, \quad \widetilde{m}=(m, x) \in M \times \mathbb{R}^{n} ;
$$

here $\lambda_{n}$ is the Lebesgue measure on $\mathbb{R}^{n}$ and $\mathcal{F}:=\left\{\mu_{m}\right\}_{m \in M}$ is the family of pointwise doubling measures on $(M, d)$, see Definition 1.11.

It is easy to show that the $M_{n}$ equipped with the family $\mathcal{F}_{n}$ is of pointwise homogeneous type but we need qualitative estimates of its basic parameters in terms of those for $(M, d)$.

This goal will be achieved in several lemmas presented below. In their formulations, $D_{n}$ and $C_{n}(t)$ are the doubling and consistency constants and $D_{n}(l)$ is the dilation function for $\left(M_{n}, d_{n}\right)$. The function $D_{n}(l)$ is defined as in (3.17) with $\mu_{m}$ replaced by measure (3.21). We recall also that $D$ and $C(t)$ are the analogous constants for $(M, d)$. 
Lemma 3.3 Assume that $n$ is related to the doubling constant $D$ by

$$
n \geq\left[\log _{2} D\right]+5 .
$$

Then we have

$$
D_{n}(1+1 / n) \leq \frac{6}{5} e^{4} .
$$

Proof. Note that the open ball $B_{R}(\widetilde{m})$ of $M_{n}$ is the set

$$
\left\{\left(m^{\prime}, y\right) \in M \times l_{1}^{n}: d\left(m^{\prime}, m\right)+\|x-y\|_{1}<R\right\} .
$$

Therefore an application of Fubini's theorem yields

$$
\mu_{\widetilde{m}}\left(B_{R}(\widetilde{m})\right)=\gamma_{n} \int_{B_{R}(m)}\left(R-d\left(m, m^{\prime}\right)\right)^{n} d \mu_{m}\left(m^{\prime}\right)
$$

here $B_{R}(m)$ is a ball of $M$ and $\gamma_{n}$ is the volume of the unit $l_{1}^{n}$-ball.

We estimate this measure with $R$ replaced by $R_{n}:=(1+1 / n) R$. Split the integral in (3.23) into one over $B_{3 R / 4}(m)$ and one over the remaining part $B_{R_{n}}(m) \backslash B_{3 R / 4}(m)$. Denote these integrals by $I_{1}$ and $I_{2}$. For $I_{2}$ we get from $(3.23)$

$$
I_{2} \leq \gamma_{n}\left(R_{n}-3 R / 4\right)^{n} \int_{B_{R_{n}}(m)} d \mu_{m}\left(m^{\prime}\right)=\gamma_{n}\left(\frac{1}{4}+\frac{1}{n}\right)^{n} R^{n} \mu_{m}\left(B_{R_{n}}(m)\right) .
$$

Using the doubling constant for $\mathcal{F}=\left\{\mu_{m}\right\}$ we further have

$$
\mu_{m}\left(B_{R_{n}}(m)\right) \leq D \mu_{m}\left(B_{R_{n} / 2}(m)\right) .
$$

Moreover, by (3.22), $D<2^{\left[\log _{2} D\right]+1} \leq \frac{1}{16} 2^{n}$. Combining all these inequalities we obtain

$$
I_{2} \leq \gamma_{n} \frac{1}{16} 2^{-n}\left(1+\frac{4}{n}\right)^{n} R^{n} \mu_{m}\left(B_{R_{n} / 2}(m)\right)
$$

To estimate $I_{1}$ we present its integrand (which equals to that in (3.23) with $R$ replaced by $R_{n}$ ) in the following way.

$$
\left(1+\frac{1}{n}\right)^{n}\left(R-d\left(m, m^{\prime}\right)\right)^{n}\left(1+\frac{d\left(m, m^{\prime}\right)}{(n+1)\left(R-d\left(m, m^{\prime}\right)\right)}\right)^{n} .
$$

Since $d\left(m, m^{\prime}\right) \leq 3 R / 4$ for $m^{\prime} \in B_{3 R / 4}(m)$, the last factor is at most $\left(1+\frac{3}{n+1}\right)^{n}$. Hence, we have

$$
I_{1} \leq \gamma_{n}\left(1+\frac{1}{n}\right)^{n}\left(1+\frac{3}{n+1}\right)^{n} \int_{B_{3 R / 4}(m)}\left(R-d\left(m, m^{\prime}\right)\right)^{n} d \mu_{m}\left(m^{\prime}\right) .
$$


Using then (3.23) we, finally, obtain

$$
I_{1} \leq e^{4} \mu_{\widetilde{m}}\left(B_{R}(\widetilde{m})\right)
$$

To estimate $D_{n}(l)$ with $l=1+1 / n$ it remains to bound the fractions

$$
\widetilde{I}_{k}:=\frac{I_{k}}{\mu_{\widetilde{m}}\left(B_{R}(\widetilde{m})\right)}, \quad k=1,2 .
$$

For $k=2$ we estimate the denominator from below as follows. Since $R_{n}<2 R$, we bound $\mu_{\widetilde{m}}\left(B_{R}(\widetilde{m})\right)$ from below by

$$
\begin{aligned}
\gamma_{n} \int_{B_{R_{n} / 2}(m)}\left(R-d\left(m, m^{\prime}\right)\right)^{n} d \mu_{m}\left(m^{\prime}\right) & \geq \gamma_{n} 2^{-n}\left(1-\frac{1}{n}\right)^{n} R^{n} \int_{B_{R_{n} / 2}(m)} d \mu_{m}\left(m^{\prime}\right) \\
& =\gamma_{n} 2^{-n}\left(1-\frac{1}{n}\right)^{n} R^{n} \mu_{m}\left(B_{R_{n} / 2}(m)\right) .
\end{aligned}
$$

Combining this with (3.24) we get

$$
\widetilde{I}_{2} \leq \frac{1}{16}\left(1-\frac{1}{n}\right)^{-n}\left(1+\frac{4}{n}\right)^{n} .
$$

Since $\left(1-\frac{1}{n}\right)^{-n} \leq\left(1-\frac{1}{5}\right)^{-5}$ as $n \geq 5$, we finally obtain

$$
\widetilde{I}_{2} \leq \frac{1}{5} e^{4}
$$

For $\widetilde{I}_{1}$ using (3.25) one immediately has

$$
\widetilde{I}_{1} \leq e^{4} .
$$

Hence, we have

$$
D_{n}(1+1 / n) \leq \sup _{\widetilde{m}, R}\left(\widetilde{I}_{1}+\widetilde{I}_{2}\right) \leq \frac{6}{5} e^{4}
$$

Our next auxiliary result evaluates the consistency constants $C_{n}(t)$ for family $\mathcal{F}_{n}=\left\{\mu_{\tilde{m}}\right\}$ in terms of that for $\mathcal{F}:=\left\{\mu_{m}\right\}$. Recall that the latter constant is the $C$ in the inequality

$$
\left|\mu_{m_{1}}-\mu_{m_{2}}\right|\left(B_{R}\left(m_{i}\right)\right) \leq \frac{C \mu_{m_{i}}\left(B_{R}\left(m_{i}\right)\right)}{R} d\left(m_{1}, m_{2}\right)
$$

where $m_{1}, m_{2}$ are arbitrary points of $M$ and $R>0$ is such that $d\left(m_{1}, m_{2}\right) \leq R$, and $i=1,2$.

\section{Lemma 3.4}

$$
C_{n}(t) \leq\left(1+\frac{4 e}{3}\right) n C, \quad 0 \leq t \leq \frac{1}{n}
$$


Proof. Using Fubini's theorem, rewrite (3.23) in the form

$$
\mu_{\widetilde{m}}\left(B_{R}(\widetilde{m})\right)=\beta_{n} \int_{0}^{R} \mu_{m}\left(B_{s}(m)\right)(R-s)^{n-1} d s
$$

where $\beta_{n}$ is the volume of the unit sphere in $l_{1}^{n}$. Then for $d_{n}\left(\widetilde{m}_{1}, \widetilde{m}_{2}\right) \leq \frac{R}{r}$ and $i=1,2$ we have

$$
\left|\mu_{\widetilde{m}_{1}}-\mu_{\widetilde{m}_{2}}\right|\left(B_{R}\left(\widetilde{m}_{i}\right)\right) \leq \beta_{n} \int_{0}^{R}\left|\mu_{m_{1}}-\mu_{m_{2}}\right|\left(B_{s}\left(m_{i}\right)\right) \cdot(R-s)^{n-1} d s .
$$

Divide now the interval of integration into subintervals $[0, R / n]$ and $[R / n, R]$ and denote the corresponding integrals over these intervals by $I_{1}$ and $I_{2}$. It suffices to find appropriate upper bounds for $I_{k}$. Replacing $B_{s}\left(m_{i}\right)$ in $I_{1}$ by the bigger ball $B_{s+R / n}\left(m_{i}\right)$ and applying (3.26) we obtain

$$
I_{1} \leq C\left(\beta_{n} \int_{0}^{R / n} \frac{\mu_{m_{i}}\left(B_{s+R / n}\left(m_{i}\right)\right)}{s+R / n}(R-s)^{n-1} d s\right) d\left(m_{1}, m_{2}\right) .
$$

Replacing $s$ by $t=s+R / n$ we bound the expression in the brackets by

$$
\left(\beta_{n} \int_{R / n}^{2 R / n} \mu_{m_{i}}\left(B_{t}\left(m_{i}\right)\right)(R-t)^{n-1} d t\right) \max _{R / n \leq t \leq 2 R / n} \frac{(R+R / n-t)^{n-1}}{t(R-t)^{n-1}} .
$$

Since $[R / n, 2 R / n] \subset[0, R]$ and the maximum $<\frac{n}{R}\left(1+\frac{1}{n-2}\right)^{n-1}<\frac{4 e}{3} \frac{n}{R}$ for $n \geq 5$, this and (3.27) yield

$$
I_{1} \leq \frac{4 e}{3} C n \frac{\mu_{\widetilde{m}_{i}}\left(B_{R}\left(\widetilde{m}_{i}\right)\right)}{R} d\left(m_{1}, m_{2}\right) .
$$

For the second term we get from (3.26)

$$
I_{2} \leq C\left(\beta_{n} \int_{R / n}^{R} \frac{\mu_{m_{i}}\left(B_{s}\left(m_{i}\right)\right)}{s}(R-s)^{n-1} d s\right) d\left(m_{1}, m_{2}\right)
$$

and by $(3.27)$ the term in the brackets is at most $\mu_{\widetilde{m}_{i}}\left(B_{R}\left(\widetilde{m}_{i}\right)\right) \cdot \frac{n}{R}$. Hence, we have

$$
I_{2} \leq C n \frac{\mu_{\widetilde{m}_{i}}\left(B_{R}\left(\widetilde{m}_{i}\right)\right)}{R} d\left(m_{1}, m_{2}\right) .
$$

Further note that $d\left(m_{1}, m_{2}\right) \leq d_{n}\left(\widetilde{m}_{1}, \widetilde{m}_{2}\right)$. Hence, we obtain finally the inequality

$$
\left|\mu_{\widetilde{m}_{1}}-\mu_{\widetilde{m}_{2}}\right|\left(B_{R}\left(\widetilde{m}_{i}\right)\right) \leq\left(1+\frac{4 e}{3}\right) n C \frac{\mu_{\widetilde{m}_{i}}\left(B_{R}\left(\widetilde{m}_{i}\right)\right)}{R} d_{n}\left(\widetilde{m}_{1}, \widetilde{m}_{2}\right)
$$

whence $C_{n}(t) \leq\left(1+\frac{4 e}{3}\right) n C, 0 \leq t \leq \frac{1}{n}$. 
Lemma 3.5 Let

$$
A_{n}:=\frac{6}{5} e^{4} n \quad \text { and } \quad n \geq\left[\log _{2} D\right]+6 .
$$

Then for all $R_{2} \geq R_{1}>0$ and $\widetilde{m} \in M_{n}$

$$
\mu_{\widetilde{m}}\left(B_{R_{2}}(\widetilde{m})\right)-\mu_{\widetilde{m}}\left(B_{R_{1}}(\widetilde{m})\right) \leq A_{n} \frac{\mu_{\widetilde{m}}\left(B_{R_{2}}(\widetilde{m})\right)}{R_{2}}\left(R_{2}-R_{1}\right) .
$$

Proof. By definition

$$
M_{n}=M_{n-1} \times \mathbb{R} \quad \text { and } \quad \mu_{\widetilde{m}}=\mu_{\widehat{m}} \otimes \lambda_{1},
$$

where $\widehat{m} \in M_{n-1}$. Then by Fubini's theorem we have for $0<R_{1} \leq R_{2}$

$$
\begin{aligned}
\mu_{\widetilde{m}}\left(B_{R_{2}}(\widetilde{m})\right)-\mu_{\widetilde{m}}\left(B_{R_{1}}(\widetilde{m})\right) & =2 \int_{R_{1}}^{R_{2}} \mu_{\widehat{m}}\left(B_{s}(\widehat{m})\right) d s \\
& \leq \frac{2 R_{2} \mu_{\widehat{m}}\left(B_{R_{2}}(\widehat{m})\right)}{R_{2}}\left(R_{2}-R_{1}\right) .
\end{aligned}
$$

We claim that for arbitrary $l>1$ and $R>0$

$$
R \mu_{\widehat{m}}\left(B_{R}(\widehat{m})\right) \leq \frac{l D_{n-1}(l)}{2(l-1)} \mu_{\widetilde{m}}\left(B_{R}(\widetilde{m})\right) .
$$

Together with the previous inequality this will yield

$$
\mu_{\widetilde{m}}\left(B_{R_{2}}(\widetilde{m})\right)-\mu_{\widetilde{m}}\left(B_{R_{1}}(\widetilde{m})\right) \leq \frac{l D_{n-1}(l)}{l-1} \cdot \frac{\mu_{\widetilde{m}}\left(B_{R_{2}}(\widetilde{m})\right)}{R_{2}}\left(R_{2}-R_{1}\right),
$$

Finally choose here $l=1+\frac{1}{n-1}$ and use Lemma 3.3. This will give the required inequality.

Hence, it remains to establish (3.28). By the definition of $D_{n-1}(l)$ we have for $l>1$

$$
\mu_{\widetilde{m}}\left(B_{l R}(\widetilde{m})\right)=2 l \int_{0}^{R} \mu_{\widehat{m}}\left(B_{l s}(\widehat{m})\right) d s \leq l D_{n-1}(l) \mu_{\widetilde{m}}\left(B_{R}(\widetilde{m})\right) .
$$

On the other hand, replacing $[0, R]$ by $\left[l^{-1} R, R\right]$ we also have

$$
\mu_{\widetilde{m}}\left(B_{l R}(\widetilde{m})\right) \geq 2 l \mu_{\widehat{m}}\left(B_{R}(\widehat{m})\right)\left(R-l^{-1} R\right)=2(l-1) R \mu_{\widehat{m}}\left(B_{R}(\widehat{m})\right) .
$$

Combining the last two inequalities we get (3.28). 


\section{Bound for the norm of the extension operator}

Let $\widehat{E}$ be the extension operator defined by $(3.14)-(3.15)$ with $(M, d, \mathcal{F})$ replaced by $\left(M_{n}, d_{n}, \mathcal{F}_{n}\right)$. To formulate the basic result we set

$$
K_{n}(l):=42\left(A_{n}+\widehat{C}_{n}\right) D_{n}(l)(l+3)
$$

where $\widehat{C}_{n}:=C_{n}(1 / n)$ and $l$ and $n$ are related by

$$
l=1+\frac{1}{n} .
$$

Proposition 3.6 The following estimate

$$
\|\widehat{E}\| \leq 56 A_{n}+\max \left(\frac{14(l+3)}{l-1}, K_{n}(l)\right)
$$

is true.

Before beginning the proof let us note that choosing here

$$
n:=\left[\log _{2} D\right]+6
$$

and applying Lemmas 3.3-3.5 we immediately obtain the inequality

$$
\|\widehat{E}\| \leq a_{0}\left(C+a_{1}\right)\left(\log _{2} D+6\right)
$$

with some numerical constants $a_{0}$ and $a_{1}$. This clearly proves Theorem 1.14 for $N=1$.

Proof. We have to show that for every $\widetilde{m}_{1}, \widetilde{m}_{2} \in M_{n}$

$$
\left\|(\widehat{E} f)\left(\widetilde{m}_{1}\right)-(\widehat{E} f)\left(\widetilde{m}_{2}\right)\right\|_{X} \leq K\|f\|_{L i p(S, X)} d_{n}\left(\widetilde{m}_{1}, \widetilde{m}_{2}\right)
$$

where $S \subset M_{n}$ and $K$ is the constant in the inequality of the proposition. It suffices to consider only two cases:

(a) $\widetilde{m}_{1} \in S$ and $\widetilde{m}_{2} \notin S$;

(b) $\widetilde{m}_{1}, \widetilde{m}_{2} \notin S$.

We assume without loss of generality that

$$
\|f\|_{\operatorname{Lip}(S, X)}=1
$$

and simplify the computations by introducing for $1 \leq i, j \leq 2$ the following notations:

$$
R_{i}:=d_{n}\left(\widetilde{m}_{i}\right), \mu_{i}:=\mu_{\tilde{m}_{i}}, B_{i j}:=B_{R_{j}}\left(\widetilde{m}_{i}\right), v_{i j}:=\mu_{i}\left(B_{i j}\right) .
$$


We assume also for definiteness that

$$
0<R_{1} \leq R_{2} .
$$

By the triangle inequality we then have

$$
0 \leq R_{2}-R_{1} \leq d_{n}\left(\widetilde{m}_{1}, \widetilde{m}_{2}\right)
$$

Further, the quantities introduced satisfy the following inequalities:

$$
\begin{aligned}
v_{i 2}-v_{i 1} & \leq \frac{A_{n} v_{i 2}}{R_{2}}\left(R_{2}-R_{1}\right), \\
\left|\mu_{1}-\mu_{2}\right|\left(B_{i j}\right) & \leq \frac{\widehat{C}_{n} v_{i j}}{R_{j}} d_{n}\left(\widetilde{m}_{1}, \widetilde{m}_{2}\right), \quad d_{n}\left(\widetilde{m}_{1}, \widetilde{m}_{2}\right) \leq \frac{R}{n} .
\end{aligned}
$$

Now, from inequality (3.8) applied to our setting and the triangle inequality we obtain

$$
\max \left\{\|\tilde{f}(\widetilde{m})\|_{X}: \tilde{m} \in B_{i 2}\right\} \leq 28 R_{2}+7(i-1) d_{n}\left(\widetilde{m}_{1}, \widetilde{m}_{2}\right) .
$$

here $i=1,2$ and we set

$$
\widetilde{f}(\widetilde{m}):=\widehat{f}(\widetilde{m})-\widehat{f}\left(\widetilde{m}_{1}\right)
$$

where $\widehat{f}$ is the extension of $f$ given by (3.7).

We now prove (3.32) for $\widetilde{m}_{1} \in S$ and $\widetilde{m}_{2} \notin S$. We begin with the evident inequality

$$
\left\|(\widehat{E} f)\left(\widetilde{m}_{2}\right)-(\widehat{E} f)\left(\widetilde{m}_{1}\right)\right\|_{X}=\frac{1}{v_{22}}\left\|\int_{B_{22}} \widetilde{f}(\widetilde{m}) d \mu_{2}\right\|_{X} \leq \max _{B_{22}}\|\widetilde{f}\|_{X},
$$

see (3.34) and (3.40). Applying (3.39) with $i=2$ we then bound this maximum by $28 R_{2}+7 d_{n}\left(\widetilde{m}_{1}, \widetilde{m}_{2}\right)$. But $\widetilde{m}_{1} \in S$ and so

$$
R_{2}=d_{n}\left(\widetilde{m}_{2}\right) \leq d_{n}\left(\widetilde{m}_{1}, \widetilde{m}_{2}\right)
$$

therefore (3.32) holds in this case with $K=35$.

The remaining case $\widetilde{m}_{1}, \widetilde{m}_{2} \notin S$ requires some additional auxiliary results. For their formulations we first write

$$
(\widehat{E} f)\left(\widetilde{m}_{1}\right)-(\widehat{E} f)\left(\widetilde{m}_{2}\right):=D_{1}+D_{2}
$$

where

$$
\begin{aligned}
& D_{1}:=I\left(\widetilde{f} ; \widetilde{m}_{1}, R_{1}\right)-I\left(\tilde{f} ; \widetilde{m}_{1}, R_{2}\right) \\
& D_{2}:=I\left(\widetilde{f} ; \widetilde{m}_{1}, R_{2}\right)-I\left(\widetilde{f} ; \widetilde{m}_{2}, R_{2}\right),
\end{aligned}
$$

see (3.16) and (3.40). 
Lemma 3.7 We have

$$
\left\|D_{1}\right\|_{X} \leq 56 A_{n} d_{n}\left(\widetilde{m}_{1}, \widetilde{m}_{2}\right)
$$

Recall that $A_{n}$ is the constant in Lemma 3.5.

Proof. By (3.42), (3.40) and (3.34),

$D_{1}=\frac{1}{v_{11}} \int_{B_{11}} \tilde{f} d \mu_{1}-\frac{1}{v_{12}} \int_{B_{12}} \tilde{f} d \mu_{1}=\left(\frac{1}{v_{11}}-\frac{1}{v_{12}}\right) \int_{B_{11}} \tilde{f} d \mu_{1}-\frac{1}{v_{12}} \int_{B_{12} \backslash B_{11}} \tilde{f} d \mu_{1}$.

This immediately implies that

$$
\left\|D_{1}\right\|_{X} \leq 2 \cdot \frac{v_{12}-v_{11}}{v_{12}} \cdot \max _{B_{12}}\|\widetilde{f}\|_{X}
$$

Applying now (3.37) and (3.36), and then (3.39) with $i=1$ we get the desired estimate.

To obtain a similar estimate for $D_{2}$ we will use the following two facts.

Lemma 3.8 Assume that for a given $l>1$

$$
d_{n}\left(\widetilde{m}_{1}, \widetilde{m}_{2}\right) \leq(l-1) R_{2}
$$

Let for definiteness

$$
v_{22} \leq v_{12}
$$

Then we have

$$
\mu_{2}\left(B_{12} \Delta B_{22}\right) \leq 2\left(A_{n}+\widehat{C}_{n}\right) D_{n}(l) \frac{v_{12}}{R_{2}} d_{n}\left(\widetilde{m}_{1}, \widetilde{m}_{2}\right)
$$

(here $\Delta$ denotes symmetric difference of sets).

Proof. Set

$$
R:=R_{2}+d_{n}\left(\widetilde{m}_{1}, \widetilde{m}_{2}\right) .
$$

Then $B_{12} \cup B_{22} \subset B_{R}\left(\widetilde{m}_{1}\right) \cap B_{R}\left(\widetilde{m}_{2}\right)$ and

(3.46) $\mu_{2}\left(B_{12} \Delta B_{22}\right) \leq\left(\mu_{2}\left(B_{R}\left(\widetilde{m}_{1}\right)\right)-\mu_{2}\left(B_{12}\right)\right)+\left(\mu_{2}\left(B_{R}\left(\widetilde{m}_{2}\right)\right)-\mu_{2}\left(B_{22}\right)\right)$.

The first term on the right-hand side is at most

$$
\left|\mu_{2}-\mu_{1}\right|\left(B_{R}\left(\widetilde{m}_{1}\right)\right)+\left|\mu_{2}-\mu_{1}\right|\left(B_{R_{2}}\left(\widetilde{m}_{1}\right)\right)+\left(\mu_{1}\left(B_{R}\left(\widetilde{m}_{1}\right)\right)-\mu_{1}\left(B_{R_{2}}\left(\widetilde{m}_{1}\right)\right) .\right.
$$


Estimating the first two terms by the inequality for the consistency constant (see Definition 1.11) and the third by Lemma 3.5 we bound this sum by

$$
\widehat{C}_{n}\left(\frac{\mu_{1}\left(B_{R}\left(\widetilde{m}_{1}\right)\right)}{R}+\frac{\mu_{1}\left(B_{R_{2}}\left(\widetilde{m}_{1}\right)\right)}{R_{2}}\right) d_{n}\left(\widetilde{m}_{1}, \widetilde{m}_{2}\right)+A_{n} \frac{\mu_{1}\left(B_{R}\left(\widetilde{m}_{1}\right)\right)}{R}\left(R-R_{2}\right) .
$$

Moreover, $R_{2} \leq R \leq l R_{2}$ and $R-R_{2}:=d_{n}\left(\widetilde{m}_{1}, \widetilde{m}_{2}\right)$, see (3.43); taking into account (3.17) for $\left(M_{n}, d_{n}\right)$ and the notations (3.34) we therefore have

$$
\mu_{2}\left(B_{R}\left(\widetilde{m}_{1}\right)\right)-\mu_{2}\left(B_{12}\right) \leq\left[\widehat{C}_{n}\left(D_{n}(l)+1\right)+A_{n} D_{n}(l)\right] \frac{v_{12}}{R_{2}} d_{n}\left(\widetilde{m}_{1}, \widetilde{m}_{2}\right) .
$$

Similarly, by Lemma 3.5 and (3.44)

$$
\begin{aligned}
& \mu_{2}\left(B_{R}\left(\widetilde{m}_{2}\right)\right)-\mu_{2}\left(B_{22}\right) \leq A_{n} \frac{\mu_{2}\left(B_{R}\left(\widetilde{m}_{2}\right)\right)}{R}\left(R-R_{2}\right) \\
\leq & A_{n} D_{n}(l) \frac{v_{22}}{R_{2}} d_{n}\left(\widetilde{m}_{1}, \widetilde{m}_{2}\right) \leq A_{n} D_{n}(l) \frac{v_{12}}{R_{2}} d_{n}\left(\widetilde{m}_{1}, \widetilde{m}_{2}\right) .
\end{aligned}
$$

Combining the last two estimates with (3.46) we get the result.

Lemma 3.9 Under the assumptions of the previous lemma we have

$$
v_{12}-v_{22} \leq 3\left(A_{n}+\widehat{C}_{n}\right) D_{n}(l) \frac{v_{12}}{R_{2}} d_{n}\left(\widetilde{m}_{1}, \widetilde{m}_{2}\right) .
$$

Proof. By (3.34) the left-hand side is bounded by

$$
\left|\mu_{1}\left(B_{12}\right)-\mu_{2}\left(B_{12}\right)\right|+\mu_{2}\left(B_{12} \Delta B_{22}\right) .
$$

Estimating these terms by (3.38) and (3.45) we get the result.

We now estimate $D_{2}$ from (3.42) beginning with

Lemma 3.10 Under the conditions of Lemma 3.8 we have

$$
\left\|D_{2}\right\|_{X} \leq K_{n}(l) d_{n}\left(\widetilde{m}_{1}, \widetilde{m}_{2}\right)
$$

where $K_{n}(l):=42\left(A_{n}+\widehat{C}_{n}\right) D_{n}(l)(l+3)$.

Proof. By the definition of $D_{2}$ and our notation, see (3.42),(3.40) and (3.34),

$$
\begin{aligned}
& \left\|D_{2}\right\|_{X}:=\left\|\frac{1}{v_{12}} \int_{B_{12}} \tilde{f} d \mu_{1}-\frac{1}{v_{22}} \int_{B_{22}} \tilde{f} d \mu_{2}\right\|_{X} \\
& \leq \frac{1}{v_{12}} \int_{B_{12}}\|\tilde{f}\|_{X} d\left|\mu_{1}-\mu_{2}\right|+\frac{1}{v_{12}} \int_{B_{12} \Delta B_{22}}\|\tilde{f}\|_{X} d \mu_{2}+\left|\frac{1}{v_{12}}-\frac{1}{v_{22}}\right| \int_{B_{22}}\|\tilde{f}\|_{X} d \mu_{2} \\
& \quad:=J_{1}+J_{2}+J_{3} .
\end{aligned}
$$


By (3.38) and (3.39) with $i=1$

$$
J_{1} \leq \frac{1}{v_{12}}\left|\mu_{1}-\mu_{2}\right|\left(B_{12}\right) \sup _{B_{12}}\|\widetilde{f}\|_{X} \leq \frac{\widehat{C}_{n}}{R_{2}} d_{n}\left(\widetilde{m}_{1}, \widetilde{m}_{2}\right) 28 R_{2}=28 \widehat{C}_{n} d_{n}\left(\widetilde{m}_{1}, \widetilde{m}_{2}\right) .
$$

In turn, by (3.45), (3.43) and (3.39)

$$
\begin{aligned}
J_{2} & \leq \frac{1}{v_{12}} \mu_{2}\left(B_{12} \Delta B_{22}\right) \sup _{B_{12} \Delta B_{22}}\|\tilde{f}\|_{X} \\
& \leq \frac{2\left(A_{n}+\widehat{C}_{n}\right) D_{n}(l)}{R_{2}} d_{n}\left(\widetilde{m}_{1}, \widetilde{m}_{2}\right)\left(7 d_{n}\left(\widetilde{m}_{1}, \widetilde{m}_{2}\right)+28 R_{2}\right) \\
& \leq 14\left(A_{n}+\widehat{C}_{n}\right) D_{n}(l)(l+3) d_{n}\left(\widetilde{m}_{1}, \widetilde{m}_{2}\right) .
\end{aligned}
$$

Finally, (3.47), (3.39) and (3.43) yield

$$
J_{3} \leq 21\left(A_{n}+\widehat{C}_{n}\right) D_{n}(l)(l+3) d_{n}\left(\widetilde{m}_{1}, \widetilde{m}_{2}\right) .
$$

Combining these we get the required estimate.

It remains to consider the case of $\widetilde{m}_{1}, \widetilde{m}_{2} \in M_{n}$ satisfying the inequality

$$
d_{n}\left(\widetilde{m}_{1}, \widetilde{m}_{2}\right)>(l-1) R_{2}
$$

converse to (3.43). Now the definition (3.42) of $D_{2}$ and (3.39) imply that

$$
\begin{aligned}
\left\|D_{2}\right\|_{X} & \leq 2 \sup _{B_{12} \cup B_{22}}\|\tilde{f}\|_{X} \\
& \leq 2\left(28 R_{2}+7 d_{n}\left(\widetilde{m}_{1}, \widetilde{m}_{2}\right)\right) \leq\left(\frac{56}{l-1}+14\right) d_{n}\left(\widetilde{m}_{1}, \widetilde{m}_{2}\right) .
\end{aligned}
$$

Combining this with the inequalities of Lemmas 3.7 and 3.10 and equality (3.41) we obtain the required estimate of the Lipschitz norm of the extension operator $\widehat{E}$. Actually, we have proved that

$$
\|\widehat{E}\| \leq 56 A_{n}+\max \left(\frac{14(l+3)}{l-1}, K_{n}(l)\right)
$$

where $K_{n}(l)$ is the constant in (3.29). This gives the proof of Theorem 1.14 for $N=1$.

Remark 3.11 Let us note that in the proof of this part of Theorem 1.14 the condition of $K$-uniformity was not used. 


\section{Proof of Theorem 1.14 for an arbitrary $N$}

(1) Let, first, $p=\infty$. Since the metric in $\left(M, d_{\infty}\right)$ is given by

$$
d_{\infty}:=\max _{1 \leq i \leq N} d_{i}
$$

where $d_{i}$ is the metric on $M_{i}$, the ball $B_{R}(m)$ of $M$ is the product of balls $B_{R}\left(m_{i}\right)$ of $M_{i}, 1 \leq i \leq N$. Therefore for the family of measures $\left\{\mu_{m}\right\}_{m \in M}$ given by the tensor product

$$
\mu_{m}:=\bigotimes_{i=1}^{N} \mu_{m_{i}}^{i}, \quad m=\left(m_{1}, \ldots, m_{N}\right)
$$

we get

$$
\mu_{m}\left(B_{R}(m)\right)=\prod_{i=1}^{N} \mu_{m_{i}}^{i}\left(B_{R}\left(m_{i}\right)\right) .
$$

Hence for the dilation function (3.17) of the family $\left\{\mu_{m}\right\}_{m \in M}$ we get

$$
D(l)=\prod_{i=1}^{N} D_{i}(l)
$$

where $D_{i}$ is the dilation function of $\left\{\mu_{m}^{i}\right\}_{m \in M_{i}}$. In particular, $\left\{\mu_{m}\right\}_{m \in M}$ satisfies the uniform doubling condition of Definition 1.11 with $\widetilde{D}:=D_{1} \cdots D_{N}$.

We check that condition (ii) of this definition (i.e., consistency with the metric) holds for this family with constant

$$
\widetilde{C}_{\infty}:=\left(\prod_{i=1}^{N} K_{i}\right) \sum_{i=1}^{N} C_{i}
$$

In fact, the identity

$$
\mu_{m}-\mu_{\widetilde{m}}=\sum_{i=1}^{N}\left(\otimes_{j=1}^{i-1} \mu_{\widetilde{m}_{j}}^{j}\right) \otimes\left(\mu_{m_{i}}^{i}-\mu_{\widetilde{m}_{i}}^{i}\right) \otimes\left(\otimes_{j=i+1}^{N} \mu_{m_{j}}^{j}\right)
$$

together with (3.50), the consistency with the metric for each $M_{j}$ and $K_{j}$ uniformity of $\left\{\mu_{m}^{j}\right\}_{m \in M_{j}}$ implies that for $\widehat{m}=m$ or $\widetilde{m}, d_{\infty}(m, \widetilde{m}) \leq R$,

$$
\begin{aligned}
\left|\mu_{m}-\mu_{\widetilde{m}}\right|\left(B_{R}(\widehat{m})\right) & \leq \sum_{i=1}^{N}\left(\prod_{j \neq i} K_{j}\right) C_{i} \frac{\mu_{\widehat{m}}\left(B_{R}(\widehat{m})\right)}{R} d_{i}\left(m_{i}, \widetilde{m}_{i}\right) \\
& \leq \widetilde{C}_{\infty} \frac{\mu_{\widehat{m}}\left(B_{R}(\widehat{m})\right)}{R} d_{\infty}(m, \widetilde{m})
\end{aligned}
$$


Thus $\left(M, d_{\infty}\right)$ is of pointwise homogeneous type with respect to the family (3.49) with optimal constants bounded by $\widetilde{D}$ and $\widetilde{C}_{\infty}$. Hence, by the previous part of the theorem for $N=1$ we have the required estimate for $\lambda(S, M ; X)$ in this case.

(2) Let now $1 \leq p<\infty$. In this case we cannot estimate the optimal constants $C$ and $D$ for the space

$$
\left(M, d_{p}\right):=\bigoplus_{p}\left\{\left(M_{i}, d_{i}\right)\right\}_{1 \leq i \leq N}
$$

directly. To overcome this difficulty we use the argument of the proof of the previous part of Theorem 1.14 (for $N=1$ ) and isometrically embed this space into the space

$$
(\widehat{M}, \widehat{d}):=\left(M, d_{p}\right) \bigoplus_{1} l_{1}^{a}
$$

with a suitable $a$. Hence, a point $\widehat{m} \in \widehat{M}$ is an $(N+a)$-tuple

$$
\widehat{m}:=(m, x):=\left(m_{1}, \ldots, m_{N}, x_{1}, \ldots, x_{a}\right)
$$

with $m \in \prod_{i=1}^{N} M_{i}$ and $x \in \mathbb{R}^{a}$. Moreover, the metric $\widehat{d}$ is given by

$$
\widehat{d}\left(\widehat{m}, \widehat{m}^{\prime}\right):=\left(\sum_{i=1}^{N} d_{i}\left(m_{i}, m_{i}^{\prime}\right)^{p}\right)^{1 / p}+\sum_{i=1}^{a}\left|x_{i}-x_{i}^{\prime}\right| .
$$

We endow $\widehat{M}$ with the family of measures given by the tensor product

$$
\mu_{\widehat{m}}:=\mu_{m} \otimes \lambda_{a}, \quad \widehat{m} \in \widehat{M},
$$

where $\lambda_{a}$ is the Lebesgue measure on $\mathbb{R}^{a}$ and $\mu_{m}:=\otimes_{i=1}^{N} \mu_{m_{i}}^{i}$.

We will show that $\lambda(S, \widehat{M} ; X)$ is bounded as required in Theorem 1.14. This immediately yields the desired estimate for $\lambda(S, M ; X)$ and completes the proof of the theorem.

To accomplish this we need

Lemma 3.12 The optimal uniform doubling constant $D$ of the family $\left\{\mu_{m}\right\}_{m \in M}$ satisfies

$$
D \leq \prod_{i=1}^{N} D_{i}
$$

Recall that $D_{i}$ is the optimal uniform doubling constant of $\left\{\mu_{m_{i}}^{i}\right\}_{m_{i} \in M_{i}}$. 
Proof. (by induction on $N$ ). For the $\mu_{m}$-measure of the ball

$$
B_{2 R}(m):=\left\{m^{\prime} \in M: \sum_{i=1}^{N} d_{i}\left(m_{i}, m_{i}^{\prime}\right)^{p}<(2 R)^{p}\right\}
$$

we get by Fubini's theorem:

$$
\mu_{m}\left(B_{2 R}(m)\right)=\int_{d^{1}<(2 R)^{p}} d \mu^{1}\left(m^{\prime}\right) \int_{d_{1}<(2 R)^{p}-d^{1}} d \mu_{1}\left(m_{1}^{\prime}\right) .
$$

Here we set for simplicity:

$$
d^{1}:=\sum_{i=2}^{N} d_{i}\left(m_{i}, m_{i}^{\prime}\right)^{p}, \quad d_{1}:=d\left(m_{1}, m_{1}^{\prime}\right)^{p}, \quad \mu^{1}:=\bigotimes_{i=2}^{N} \mu_{m_{i}}^{i}, \quad \mu_{1}:=\mu_{m_{1}}^{1} .
$$

The second integral is the $\mu_{1}$-measure of the ball $B_{2 \rho}\left(m_{1}\right)$ where $\rho:=$ $\sqrt[p]{R^{p}-2^{-p} d^{1}}$ which is bounded by $D_{1} \mu_{1}\left(B_{\rho}\left(m_{1}\right)\right)$. This and Fubini's theorem imply that

$$
\begin{aligned}
\mu_{m}\left(B_{2 R}(m)\right) & \leq D_{1} \int_{2^{-p} d^{1}<R^{p}} d \mu^{1}\left(m^{\prime}\right) \int_{d_{1}<R^{p}-2^{-p} d^{1}} d \mu_{1}\left(m_{1}^{\prime}\right) \\
& =D_{1} \int_{d_{1}<R^{p}} d \mu_{1}\left(m_{1}^{\prime}\right) \int_{d^{1}<(2 R)^{p}-2^{p} d_{1}} d \mu^{1}\left(m^{\prime}\right) .
\end{aligned}
$$

By the induction hypothesis the inner integral in the right-hand side is bounded by

$$
\left(\prod_{i=2}^{N} D_{i}\right) \mu^{1}\left(B_{\sqrt[p]{R^{p}-d_{1}}}\left(m_{2}, \ldots, m_{N}\right)\right)=\prod_{i=2}^{N} D_{i} \int_{d^{1}<R^{p}-d_{1}} d \mu^{1}\left(m^{\prime}\right) .
$$

Combining this with the previous inequality to get the required result:

$$
\mu_{m}\left(B_{2 R}(m)\right) \leq\left(\prod_{i=1}^{N} D_{i}\right) \mu_{m}\left(B_{R}(m)\right) .
$$

Using Lemma 3.12 we estimate now the dilation function $D_{a}(s)$ of the family $\left\{\mu_{\widehat{m}}\right\}$. Recall that for $s>1$

$$
D_{a}(s):=\sup _{\widehat{m} \in \widehat{M}}\left\{\frac{\mu_{\widehat{m}}\left(B_{s R}(\widehat{m})\right)}{\mu_{\widehat{m}}\left(B_{R}(\widehat{m})\right)}\right\} .
$$

To this end we simply apply to this setting Lemma 3.3 with $D$ replaced by $\prod_{i=1}^{N} D_{i}$ and $n$ by $a$. This yields 
Lemma 3.13 If $a \geq\left[\log _{2} \prod_{i=1}^{N} D_{i}\right]+5$, then

$$
D_{a}(1+1 / a) \leq \frac{6}{5} e^{4} .
$$

Now we estimate the consistency constant $\widetilde{C}_{a}:=C(1 / a)$ for the family $\left\{\mu_{\widehat{m}}\right\}_{\widehat{m} \in \widehat{M}}$, see Definition 1.11. To this goal we use (3.53) for $\mu_{\widehat{m}}-\mu_{\widehat{m}^{\prime}}$, $\widehat{d}\left(\widehat{m}, \widehat{m}^{\prime}\right) \leq \frac{R}{a}$, and then apply Fubini's theorem to get for $\widehat{m}^{\prime \prime}:=\widehat{m}$ or $\widehat{m}^{\prime}$ $\left|\mu_{\widehat{m}}-\mu_{\widehat{m}^{\prime}}\right|\left(B_{R}\left(\widehat{m}^{\prime \prime}\right)\right)$

$$
\leq \sum_{i=1}^{N} \int_{\delta_{a}<R} d \lambda_{a} \int_{d^{i}<\left(R-\delta_{a}\right)^{p}} d \mu_{i}^{\prime} d \mu_{i} \int_{d_{i}<\left(R-\delta_{a}\right)^{p}-d^{i}} d\left|\mu_{m_{i}}^{i}-\mu_{m_{i}^{\prime}}^{i}\right| .
$$

Here we use the notation:

$$
\begin{gathered}
\delta_{a}:=\sum_{j=1}^{a}\left|x_{j}-x_{j}^{\prime \prime}\right|, \quad d^{i}:=\sum_{j \neq i} d_{j}\left(m_{j}^{\prime \prime}, m_{j}\right)^{p}, \quad d_{i}:=d\left(m_{i}^{\prime \prime}, m_{i}\right)^{p}, \\
\mu_{i}^{\prime}:=\bigotimes_{j<i} \mu_{m_{j}^{\prime}}^{j}, \quad \mu_{i}:=\bigotimes_{j>i} \mu_{m_{j}}^{j} .
\end{gathered}
$$

Recall that $\widehat{m}=(m, x) \in M \times \mathbb{R}^{a}$.

The inner integral in the $i$-th term of the right-hand side of (3.56) equals $\left|\mu_{m_{i}}^{i}-\mu_{m_{i}^{\prime}}^{i}\right|\left(B_{\rho}\left(m_{i}^{\prime \prime}\right)\right)$ where

$$
\rho:=\sqrt[p]{\left(R-\delta_{a}\right)^{p}-d^{i}}
$$

Replacing here $\rho$ by

$$
\rho_{a}:=\sqrt[p]{\left(R_{a}-\delta_{a}\right)^{p}-d^{i}}
$$

with $R_{a}:=\left(1+\frac{1}{a}\right) R$ and applying the consistency inequality for $\left(M_{i}, d_{i}\right)$ (observe that $\left.d_{i}\left(m_{i}, m_{i}^{\prime}\right) \leq \frac{R}{a}\right)$ we then bound this inner integral by

$$
\frac{C_{i} \mu_{m_{i}^{\prime \prime}}^{i}\left(B_{\rho_{a}}\left(m_{i}^{\prime \prime}\right)\right)}{\rho_{a}} d_{i}\left(m_{i}, m_{i}^{\prime}\right) .
$$

Since $d^{i} \leq\left(R-\delta_{a}\right)^{p}$, the denominator here is at least $R_{a}-R=\frac{1}{a} R$. Therefore the inner integral is bounded by

$$
\frac{a C_{i} d_{i}\left(m_{i}, m_{i}^{\prime}\right)}{R} \int_{d_{i}<\left(R_{a}-\delta_{a}\right)^{p}-d^{i}} d \mu_{m_{i}^{\prime \prime}}^{i}
$$

Inserting this in (3.56) and replacing there $R$ by $R_{a}$ we get

$$
\left|\mu_{\widehat{m}}-\mu_{\widehat{m}^{\prime}}\right|\left(B_{R}\left(\widehat{m}^{\prime \prime}\right)\right) \leq \frac{a}{R} \sum_{i=1}^{N} C_{i} d_{i}\left(m_{i}, m_{i}^{\prime}\right) \int_{B_{R_{a}}\left(\widehat{m}^{\prime \prime}\right)} d \lambda_{a} d \mu_{i}^{\prime} d \mu_{i} d \mu_{m_{i}^{\prime \prime}}^{i}
$$


To replace in this inequality each $\mu_{m_{j}^{\prime}}^{j}\left(\right.$ or $\mu_{m_{j}}^{j}$ ) by $\mu_{m_{j}^{\prime \prime}}^{j}$ we now use the $K_{j}$-uniformity of the family $\left\{\mu_{m_{j}}^{j}\right\}_{m_{j} \in M_{j}}$, see Definition 1.13. Applying this to the right-hand side of the previous inequality and recalling definition (3.55) we estimate the $i$-th integral there by

$$
\begin{aligned}
\left(\prod_{i=1}^{N} K_{i}\right) \int_{B_{R_{a}}\left(\widehat{m}^{\prime \prime}\right)} d \lambda_{a} d \mu_{m^{\prime \prime}} & =\left(\prod_{i=1}^{N} K_{i}\right) \mu_{\widehat{m}^{\prime \prime}}\left(B_{R_{a}}\left(\widehat{m}^{\prime \prime}\right)\right) \\
& \leq D_{a}(1+1 / a)\left(\prod_{i=1}^{N} K_{i}\right) \mu_{\widehat{m}^{\prime \prime}}\left(B_{R}\left(\widehat{m}^{\prime \prime}\right)\right) .
\end{aligned}
$$

Combining with the previous inequality we get for $\widehat{m}^{\prime \prime}=\widehat{m}$ or $\widehat{m}^{\prime}$

$$
\left|\mu_{\widehat{m}}-\mu_{\widehat{m}^{\prime}}\right|\left(B_{R}\left(\widehat{m}^{\prime \prime}\right)\right) \leq \frac{a D_{a}(1+1 / a)}{R}\left(\prod_{i=1}^{N} K_{i}\right)\left(\sum_{i=1}^{N} C_{i} d_{i}\left(m_{i}, m_{i}^{\prime}\right)\right) \mu_{\widehat{m}^{\prime \prime}}\left(B_{R}\left(\widehat{m}^{\prime \prime}\right)\right) .
$$

By Hölder's inequality the sum in the brackets is at most

$$
\left(\sum_{i=1}^{N} C_{i}^{q}\right)^{1 / q}\left(\sum_{i=1}^{N} d_{i}\left(m_{i}, m_{i}^{\prime}\right)^{p}\right)^{1 / p}=:\left(\sum_{i=1}^{N} C_{i}^{q}\right)^{1 / q} d_{p}\left(m, m^{\prime}\right)
$$

here $\frac{1}{p}+\frac{1}{q}=1$. Hence the consistency constant $\widehat{C}_{a}$ of the family $\left\{\mu_{\widehat{m}}\right\}_{\widehat{m} \in \widehat{M}}$ satisfies

$$
\widehat{C}_{a} \leq a D_{a}(1+1 / a)\left(\prod_{i=1}^{N} K_{i}\right)\left(\sum_{i=1}^{N} C_{i}^{q}\right)^{1 / q}
$$

Choose now $a:=\left[\log _{2} \prod_{i=1}^{N} D_{i}\right]+6$ and use Lemma 3.5 for the space $(\widehat{M}, \widehat{d})$ equipped with the family $\left\{\mu_{\widehat{m}}\right\}_{\widehat{m} \in \widehat{M}}$. Then we have

$$
A_{a} \leq \frac{6}{5} e^{4}\left(\log _{2}\left(\prod_{i=1}^{N} D_{i}\right)+6\right)
$$

Combining Lemma 3.13 with (3.57) and the above inequality we finally obtain the required result (cf. (3.48))

$$
\lambda(S, \widehat{M} ; X) \leq c_{0}\left(\widetilde{C}_{p}+1\right)\left(\log _{2}\left(\prod_{i=1}^{N} D_{i}\right)+1\right)
$$

with $\widetilde{C}_{p}:=\left(\sum_{i=1}^{N} C_{i}^{q}\right)^{1 / q}\left(\prod_{i=1}^{N} K_{i}\right)$ and $\frac{1}{p}+\frac{1}{q}=1$. 
Remark 3.14 It is easily checked that the proof of this part of Theorem 1.14 is valid for the case of $M_{1}$ a doubling metric space. In fact, due to Koniagin-Vol'berg's theorem [KV] this space can be endowed with a doubling measure $\mu$ and therefore Theorem 1.14 holds for this case with $N=1$, see Remark 3.11. If $N \geq 2$ note that since the family of doubling measures for $M_{1}$ consists of a single measure $\mu$, the condition of $K_{1}$-uniformity is not needed in the proof. Hence, in this setting Theorem 1.14 holds with $D_{1}=D(\mu), C_{1}=0$ and with 1 instead of $K_{1}$.

\section{References}

[BHK] Bonk, M., Heinonen, J. And Koskela, P.: Uniformizing Gromov hyperbolic spaces. Astérisque, 270, 2001.

[BS] Bonk, M. And Schramm, O.: Embeddings of Gromov hyperbolic spaces. Geom. Funct. Anal. 10 (2000), 266-306.

[BH] Bridson, M. And Haefliger, A.: Metric spaces of non-positive curvature. Grundlehren der Mathematischen Wissenschaften 319. SpringerVerlag, Berlin, 1999.

[BB] Brudnyi, A. And Brudnyi, Yu.: Metric spaces with linear extensions preserving Lipschitz condition. Amer. J. Math. 129 (2007), no. 1, 217-314.

[BSh] Brudnyi, Yu. and Shvartsman, P.: The Whitney problem of existence of a linear extension operator. J. Geom. Anal. 7, No. 4 (1997), 515-574.

[CW] Colfman, R. R. And Weiss, G.: Analyse harmonique non-commutative sur certains espaces homogènes. Lecture Notes in Mathematics, 242. Springer-Verlag, Berlin-New York, 1971

[CDP] Coornaert, M., Delzant, T. and Papadopoulos, A.: Géometrié et théorie de groupes. Lecture Notes in Mathematics 1441. Springer-Verlag, Berlin, 1990.

[D] Dugundji, J.: An extension of Tietze's theorem. Pacific J. Math. 1 (1951), 353-367.

[GK] Godefroy, G. and Kalton, N. J.: Lipschitz-free Banach spaces. Studia Math. 159 (2003), 121-141.

[KV] Koniagin, S. And Vol'Berg, A.: There is a homogeneous measure on any compact subset of $\mathbb{R}^{n}$. Math. USSR- Izv. 30 (1988), 629-638. (Russian).

[LPS] Lang, U., Pavlović, B. and Schroeder, V.: Extensions of Lipschitz maps into Hadamard spaces. Geom. Funct. Anal. 10 (2000), 1527-1553.

[LN] LeE, J.R. AND NAOR, A.: Extending Lipschitz functions via random metric partitions. Invent. Math. 160 (2005), 59-95.

[LS] Luukkainen, J. And Saksman, E.: Every complete doubling metric space carries a doubling measure. Proc. Amer. Math. Soc. 126 (1998), no. $2,531-534$. 
[NPSS] Naor, A., Peres, Y., Schramm, O. And Sheffield, S.: Markov chains in smooth Banach spaces and Gromov-hyperbolic metric spaces. Duke Math. J. 134 (2006), 165-197.

[N] Novikov, P. S.: Sur les fonctions implicites mesurables. B. Fund. Math. 17 (1931), 8-25.

[W] Weaver, N.: Lipschitz algebras. World Scientific Publishing, River Edge, New Jersey, 1999.

Recibido: 10 de enero de 2006

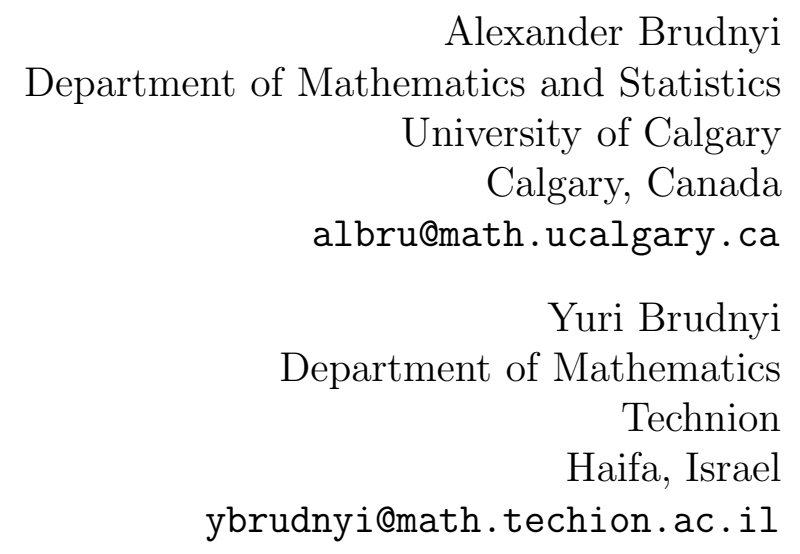

The first author is supported in part by NSERC. 